Research Article

\title{
Evacuation Model of Emotional Contagion Crowd Based on Cellular Automata
}

\author{
Qian Xiao (i) ${ }^{1}$ and Jiayang $\mathrm{Li}\left(\mathbb{D}^{1,2}\right.$ \\ ${ }^{1}$ Department of Information Engineering, Shenyang University, Shenyang 110044, China \\ ${ }^{2}$ Department of Business Administration, Northeastern University, Shenyang 110000, China \\ Correspondence should be addressed to Qian Xiao; xiaoqian_neu@163.com
}

Received 30 January 2021; Revised 7 March 2021; Accepted 13 March 2021; Published 27 March 2021

Academic Editor: Jorge E. Macias-Diaz

Copyright (c) 2021 Qian Xiao and Jiayang Li. This is an open access article distributed under the Creative Commons Attribution License, which permits unrestricted use, distribution, and reproduction in any medium, provided the original work is properly cited.

\begin{abstract}
Crowd evacuation under emergency is an important task of world public security research and practice. In order to describe the microemotional contagion of evacuation individuals, a cellular automata-based evacuation model of emotional contagion crowd based on the classical SIS model of infectious diseases is proposed in this paper. Firstly, the state of evacuation individual is defined as "emotional susceptible" and "emotional infective." Then, a dynamic model considering emotional contagion is established with cellular automata. Based on the models of static floor field and dynamic floor field, the emotion updating rules and state updating rules are constructed. The influence of perception domain radius on pedestrian evacuation process is analyzed through experiments. The conclusion can provide evacuation guidance for evacuation individuals. The comparative experiment results show that the improved model can reflect the movement characteristics of evacuation individuals effectively. The evacuation efficiency of the whole system is also effectively improved due to the consideration of emotional contagion and evacuation strategy.
\end{abstract}

\section{Introduction}

With the development of social progress and frequent economic, political, and cultural exchanges, people's activities are increasingly rich. In recent years, various accidents caused by the crowd have happened frequently. Therefore, how to grasp the characteristics of crowd evacuation behavior in emergency is the key to formulate effective guidance plan. In the past few decades, many researchers have mainly used experimental and simulation methods to study crowd evacuation behavior. The experimental methods mainly include the questionnaire method after the real event and controlled evacuation experiment with personnel participation. Afterwards, questionnaire method has information lag. Controllable experiments are often costly, and it is difficult to simulate dangerous scenarios, such as fire and terrorist attacks. Therefore, building a crowd evacuation simulation model in line with the actual situation has gradually become an important method to analyze the characteristics of crowd evacuation behavior, which has been widely used [1-3]. Simulation model method is to design reasonable building facilities and environment and reshape the basic attributes of the crowd. Then, take the crowd or individual as the research object to carry out the simulation of normal or emergency evacuation process. The evacuation simulation model can be used to evaluate the effectiveness of guidance measures, help the site management to make emergency plans, and provide theoretical support for emergency decision-making.

At present, the research of indoor crowd evacuation simulation model is mainly divided into macromodel and micromodel. The macrosimulation model usually adopts the classical graph theory method. The evacuation decisionmaking problem is transformed into an evacuation route selection problem. It usually adopts the minimum cost maximum flow method, the queuing theory method, and the shortest path method [4-7]. Micromodels usually take individuals as objects. It can identify many typical characteristics of individual movement or human evacuation behavior. Common models include cellular automata model, 
social force model, lattice gas model, and visual obstacle model [8-13]. Compared with other models, cellular automata (CA) model has powerful complex computing function and highly dynamic characteristics. It has a strong ability to simulate the spatiotemporal dynamic evolution of space complex system and requires relatively less computation. Therefore, it has been widely used in crowd evacuation research.

Cellular automata were proposed by Blue et al. [14] in 1997, and then it began to be applied in pedestrian evacuation. The basic CA model represents the environment site as a grid composed of cells. The evacuation individuals move from one cell to the adjacent cell according to certain rules. Liu et al. [15] established an improved CA evacuation model considering the influence of the density of people around the exit on the evacuation behavior. Li et al. [16] proposed an extended CA model, considering the effect of sudden hazard. Zhang et al. [17] established a multiagent decision-making framework based on CA model to study the evacuation problem of stadium with or without obstacles. Yang et al. [18] present a new static floor field method based on cellular automaton for simulations of evacuation processes. Zheng et al. [19] proposed an extended CA model to study the effect of three-dimensional smoke diffusion on crowd evacuation. Geng et al. [20] proposed an extended cellular automata model to simulate the individual evacuation information under adverse line of sight conditions with uncertainty.

In emergencies, individual emotion plays an important role in evacuation decision-making. The emotion of the evacuation individual is dynamic and can be perceived and spread. Franovetter [21] proposed the concept of "threshold theory" and proposed that every event has an emotional threshold. The OCEAN model [22] proposed by McCrae et al. is the most widely used personality model, and the model describes individual personality from five vector dimensions. Helbing [23] simulated the dynamic escape behavior of evacuating individuals and concluded that escape behavior was a behavioral manifestation of emotion and confirmed that emotion among many people was contagious. Wang et al. [24] investigated the correlations between evacuation crowd characteristics and the evacuation emotion in subway emergencies by questionnaire. Shi et al. [25] proposed a new deterministic model of impatience, which described the impatience during pedestrian evacuation qualitatively and quantitatively. Wang et al. [26] considered that emotional contagion has an impact on the behavior of both individuals and groups and formulated the control problem of emotional contagion as an optimal control problem of crowd evacuation.

The human study of group emotion communication originated from infectious diseases. There are two kinds of scientific epidemic models to explore how diseases spread among groups, such as SIR model and SIS model [27, 28]. SIR model is to study the transmission relationship among "susceptible," "infective," and "removal." Fu et al. [29] modified the epidemiological SIR model and proposed a new (CA-SIRS) emotional contagion model. The SIS model considers that it is difficult for individuals to achieve complete immunity when considering the infection of some diseases. Therefore, there are only "susceptible persons" and "infected persons" in the process of infection. The SIS model describes that an individual can change from a susceptible state to an infected state. After cure, he is still in a susceptible state and has the probability of being infected. However, the total number of infected people in SIS model should remain unchanged, and it is not suitable to describe the microemotional contagion of pedestrian evacuation individuals. Therefore, this paper combines SIS model with CA model to build a crowd evacuation model, considering emotional contagion under emergency. Then, the evacuation process is simulated.

\section{Basic Theory}

2.1. Cellular Automata Model. Cellular automata are a kind of dynamic system with discrete time and space. It uses discrete space layout and discrete time interval to divide the cell into finite states. The evolution of the individual state of the cell is only related to its current state and the state of a local neighborhood. Each cell scattered in the regular grid takes a finite discrete state, follows the same action rules, and updates synchronously according to the determined local rules. The basic elements of cellular automata are as follows:

(1) Space: the spatial lattice of cells in space can be onedimensional, two-dimensional, or multidimensional.

(2) State set: there are only two different states, which can be coded as 0 and 1 , respectively, or represented by different colors.

(3) Neighbor: there is a cell with radius of 1 around a cell, which can affect the state of the cell at the next moment. The most common neighbors are Moore type and von Neumann type.

(4) According to the state of the cell and its eight neighboring cells, the dynamic function or state transfer equation of the cell state at the next moment is determined.

2.2. Typical Models of Infectious Diseases. Emotional contagion can be studied with reference to the disease transmission model. There are two kinds of traditional infectious disease models: SIR model and SIS model.

SIR model refers to three types of individual status: "susceptible," "infective," and "removal." $S, I$, and $R$, respectively, represent the proportion of the three groups in the total group. SIR model does not calculate the new birth rate and death rate in the process of infection, so $S+I+R=1$. The model reflects the macroprocess of human infection: when infectious diseases occur, the total number of three groups of people is constant. Once "susceptible" $(S)$ contacted with "infective" $(I)$ will be infected with probability; "infective" $(I)$ will change to "removal" $(R)$ with a certain probability; "removal" is equivalent to vaccine injector and will no longer be infected with disease immunity.

The other classical SIS model considers that when some diseases are infected, it is difficult for individuals to achieve complete immunity. Therefore, there are only "susceptible" 
and "infective" in the process of infection. SIS emotional contagion model describes that an individual changes from an easily infected state to an infected state. After being cured, he is still an easily infected individual and will enter the infectious system again. The differential system dynamics equation of the model is shown in equation (1). The state transition diagram is shown in Figure 1.

$$
\begin{aligned}
S+I & =1, \\
\frac{\mathrm{d} S}{\mathrm{~d} t} & =-\beta S I+\gamma I, \\
\frac{\mathrm{d} I}{\mathrm{~d} t} & =\beta S I-\gamma I,
\end{aligned}
$$

where $\beta$ is the daily contact infection rate; $\gamma$ is the daily cure rate.

Since the SIS model adopts the macroscopic model of infection dynamics expressed by differential equation, it is not suitable for describing the microemotional contagion of evacuation individuals. In addition, the SIS model assumes that the total number of people does not change during the process of infection. However, when pedestrians evacuate, once they leave the exit, it is a safe area. That is, the number of people in the dangerous area at every moment is dynamic. Based on the above, this paper combines the classical SIS model with cellular automata to build a dynamic pedestrian evacuation model, considering emotional contagion in emergencies.

\section{Evacuation Model of Emotional Contagion Crowd Based on Cellular Automata}

3.1. Basic Cell Definition of Cellular Automata. In this paper, cellular automata model is used to simulate the evacuation situation in independent space. The space is $L$ in length and $W$ in width. It is divided into cell lattices of $0.4 \times 0.4$ for one person to stand. Each cell is represented by a set of number pairs, such as cell $(x, y)$. The distance between adjacent cells is $\mathrm{cl}=0.4 \mathrm{~m}$. The number of exits is $m$. The width is $\mathrm{el}_{i}$, where

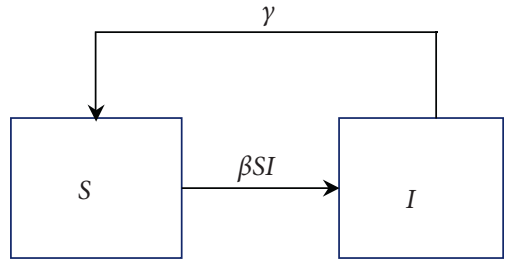

FIgURE 1: Schematic diagram of SIS model state transition.

$i \in[1,2, \ldots, m]$. When the initial state $t=0$, pedestrians are randomly distributed in the space, each occupying a cell. After the evacuation, Moore neighborhood rule with radius of 1 is adopted in the neighborhood of the cell, as shown in Figure 2. The time step is $\Delta t=0.4 s$. Each time, the pedestrians determine the next move strategy according to the transfer probability of the cell in each direction until they reach the exit and escape.

Burstedde et al. [30] introduced the concepts of static floor field and dynamic floor field in the CA model. Many scholars added friction, inertia, force, obstacle, and other factors to its expansion. The probability of pedestrian moving to its adjacent cell lattice is determined by static floor field, dynamic floor field, and their interaction. This paper analyzes the transition probability of static floor field and dynamic floor field, respectively. In this paper, the model is established on the basis of static field and dynamic field.

3.2. Static Floor Field. The static floor field (SFF) in cellular automata is the distance from each cell to the effective exit. Let $S_{j i}(t)$ denote the shortest distance from the neighborhood cell $i$ of evacuation individual $j$ to the exit at time $t$. Let the location coordinate of evacuation individual $j$ be $C(x, y)$, and the location coordinate of neighborhood cell $i$ be $C\left(x_{i}, y_{i}\right)$. There are $m$ effective exits in the evacuation space. The midpoint coordinate of each exit is $C\left(x_{e_{k}}, y_{e_{k}}\right)$. According to the static floor field of cellular automata, the transition probability formula is shown in the following equation:

$$
S_{j i}=\max _{i \in \text { all neighbors }}\left\{\min _{i \longrightarrow \text { all exit } k \in[1, m]}\left\{\left[\left(x_{e_{k}}-x_{i}\right)^{2}+\left(y_{e_{k}}-y_{i}\right)^{2}\right]^{1 / 2}\right\}\right\}-\min _{i \longrightarrow \text { all exit } k \in[1, m]}\left\{\left[\left(x_{e_{k}}-x_{i}\right)^{2}+\left(y_{e_{k}}-y_{i}\right)^{2}\right]^{1 / 2}\right\},
$$

where the second term of the formula represents the minimum value of the neighborhood cell distance among all exits. The first term represents the maximum value of the minimum value of the neighborhood cell distance among all exits. The formula is a two-level progressive relation. The first layer is to calculate the shortest distance between eight cells in the neighborhood and all effective exits. The second layer is to compare the deviation between the shortest distance of eight cells in the neighborhood and the maximum of the shortest distance. If it is equal to the maximum value of the shortest distance, then $S_{j i}(t)=0$. If the deviation from the maximum value of the shortest distance is greater, then the cell has the shortest distance from the exit. That is, the greater the $S_{j i}(t)$ value is, the greater the probability of the cell being selected.

3.3. Dynamic Floor Field. In the emergency situation, the state of the evacuation individual changes after the emotion reaches a certain threshold. Thus, it causes the change of behavior strategy. The behavior execution process of evacuation individual $j$ at time $t$ is shown in Figure 3. Therefore, the dynamic field of this model mainly includes emotion updating rules and state updating rules. 


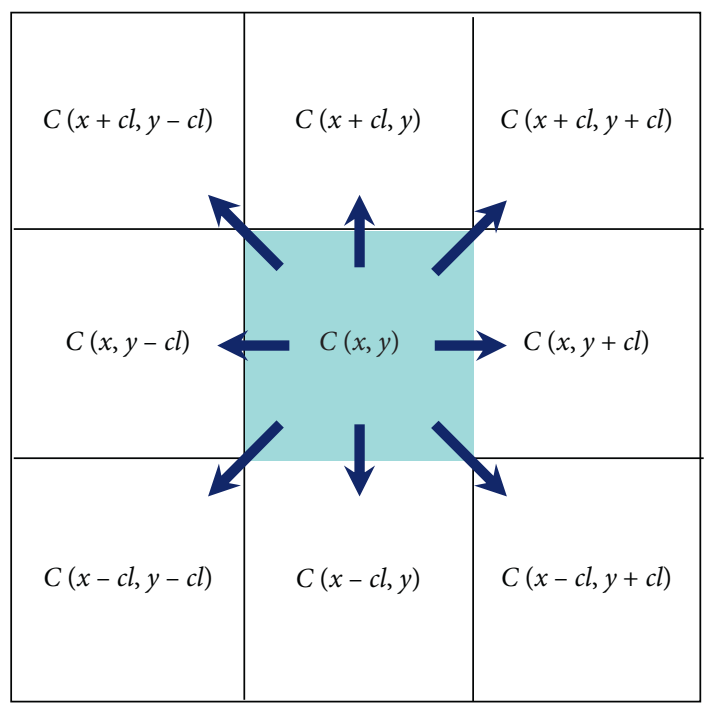

FIGURE 2: Moore neighborhood rule and direction transition probability.

3.3.1. Emotional Contagion Function. The emotional intensity of evacuation individual $j$ fluctuates with time. The dynamic equation is shown in the following equation:

$$
\mathrm{PE}_{j}(t)=\mathrm{PE}_{j}(t-1)+(1-\xi) \cdot \Delta e_{j}(t)+\tau,
$$

where $\mathrm{PE}_{j}(t-1)$ is the emotional intensity of evacuation individual $j$ at time $t-1 ; \Delta e_{j}(t)$ is the emotion increment of evacuation individual $j$ at time $t$; $\xi$ is the perceptual attenuation coefficient; and the value range is $[0,1]$. It indicates that there is a certain attenuation in the superposition of emotion increment of evacuation individuals. $\tau$ is the random emotional disturbance of individual $j$ at time $t$.

IEquation (3) shows the renewal mechanism of emotion and the evolution mechanism of infection. The emotional intensity $\mathrm{PE}_{j}(t)$ is based on the self-emotional intensity at time $t-1$ and superimposed with the emotional influence of others in the surrounding perception area.

3.3.2. Emotional Perception Increment. The emotional fluctuation of evacuation individuals is related to their own speed and the emotion of others around them. Therefore, this model defines the emotional increment function of evacuation individual $j$ at time $t$, as shown in the following equation:

$$
\Delta e_{j}(t)=\Delta f_{j}(v, t)+\Delta \varphi_{j}(t),
$$

where $\Delta f_{j}(v, t)$ is the internal emotional change of evacuation individual $j$ caused by speed at time $t ; \Delta \varphi_{j}(t)$ is the emotional change of others perceived by individual $j$ at time $t$.

(1) Definition of function $\Delta f_{j}(v, t)$ : in the process of evacuation, if the evacuation speed is slower than the maximum expected speed, the negative emotions (irritability, tension, and anxiety) increase faster. If the walking speed of the evacuation individual is close to the maximum expected speed, the growth of their bad mood is relatively

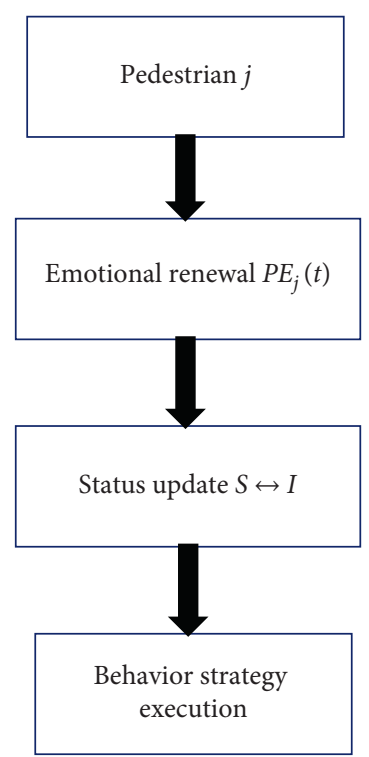

FIGURE 3: Diagram of an iterative process of evacuation individual $j$ at time $t$.

slow [24]. Therefore, the emotional increment function caused by speed is shown in the following equation:

$$
\Delta f_{j}(v, t)=\left(1-\frac{v_{j}(t-1)}{v_{j}^{\max }}\right) \cdot \exp \left(-\alpha \cdot\left(\frac{v_{j}(t-1)}{v_{j}^{\max }}\right)\right),
$$

where $v_{j}(t-1)$ represents the speed of the previous movement of the evacuation individual $j$ at time $t ; v_{j}^{\max }$ is the maximum expected speed of individual $j$ during evacuation; $\alpha$ is the velocity sensitive parameter of evacuation individual $j$ at time $t$; and the value range is $[0,1]$.

This function can clearly express the influence of the difference between the previous moving speed and the maximum expected speed on the emotional fluctuation. If the moving speed is close to the maximum expected speed, it will cause less fluctuation of individual emotion. On the contrary, if the moving speed is far less than the maximum expected speed, the $\Delta f_{j}(v, t)$ value will be close to 1 . In other words, the degree of emotion will increase.

(2) The definition of function $\Delta \varphi_{j}(t)$ : Zhang et al. [31] proposed that there were circular perception domains and perception domain radius for evacuation individuals. The traditional perception domain discretization thinks that the emotional state of each individual in the perception area can be fully perceived by the individual, but the actual situation is not so. Geng et al. [20] considered that the influence of information communication in line of sight is different from that in nonline of sight. At the same time, this model considers that the scope of emotional perception is not limited to contact perception. For individuals, the emotions of the surrounding people will be perceived, especially the emotions in the visual area will be more intense through visual perception. Therefore, this model divides the perception area into visual perception domain RV and nonvisual perception domain RI. It is considered that the 
emotion of others in the visual domain can be fully perceived, while the emotion in the nonvisual domain can be perceived through pushing, sound, action, etc., and the perception degree will decline to a certain extent.

Li et al. used angle $\pi / 4$ to represent the visual range of pedestrians in eight neighborhood directions [32]. Usually the horizontal angle of people's binocular vision is about $3 \pi / 4 . \pi / 2$ is selected as the angle of visual area in this model, considering that people's field of vision will shrink under emotional fluctuations. Then, the parameters of the visual perception domain RV and the nonvisual perception domain RI are defined as follows:

(1) $\operatorname{Cell}_{j}(x, y)$ is the location of evacuation cell $j$

(2) $\theta_{j}(t)$ is the angle radian between the forward direction $\operatorname{dir}_{j}(t-1)$ of the evacuation individual cell $j$ and the horizontal axis of the coordinate. Its value can be $(0, \pi / 4, \pi / 2,3 \pi / 4, \pi, 5 \pi / 4,6 \pi / 4,7 \pi / 4)$

(3) $\operatorname{dir}_{j}(t-1)$ is the forward direction of evacuation individual cell $j$ at the last moment. The corresponding relationship with $\theta_{j}(t)$ is shown in Figure 4

(4) The visual area of evacuation individual is a sector with $\operatorname{arc} \pi / 2$

(5) $R$ is the emotional perception domain radius of evacuation individuals

The definition of visual perception domain RV: the sector with the cell coordinate cell ${ }_{j}(x, y)$ of evacuation individual $j$ at time $t$ as the center, $\left[\theta_{j}(t)-\pi / 4, \theta_{j}(t)+\pi / 4\right]$ as the angle, and $R$ as the radius is the visual perception domain $\mathrm{RV}$ of the evacuation individual at time $t$. As shown in Figure 5, the green color area is the visual perception domain after discretization. The emotions of the evacuated individuals in this area can be perceived.

Definition of nonvisual perception domain RI: the circle with the cell coordinate cell ${ }_{j}(x, y)$ of evacuation individual $j$ at time $t$ as the center, $R$ as the radius, and removing the part of RV is the nonvisual perception domain RI of the evacuation individual at time $t$. As shown in Figure 5, the gray area is the nonvisual perception domain. The emotion of the evacuation individual is partially perceived.

Therefore, the visual perception domain and nonvisual perception domain, corresponding to different forward directions of evacuation individual cell $j$ at time $t$, are shown in Figure 6.

According to the perception domain, the evacuation space is divided into three situations: exit perception area $A_{1}$, adjacent edge area $A_{2}$, and other area $A_{3}$, as shown in Figure 7.

As shown in Figure 7, exit perception area $A_{1}$ is composed of two squares with exit endpoint as vertex and $R$ as side length and a rectangle with length $R$ and width $e l$. When evacuation individuals are in this area, they are not affected by other people's emotions, because the exit is the most attractive. Therefore, it can be considered that when in the exit perception area $A_{1}$, the emotion of the evacuation individual no longer fluctuates, such as the location of $n_{1}$. The perception area of evacuation individuals in the adjacent edge area $A_{2}$ is an incomplete circle, which needs to be

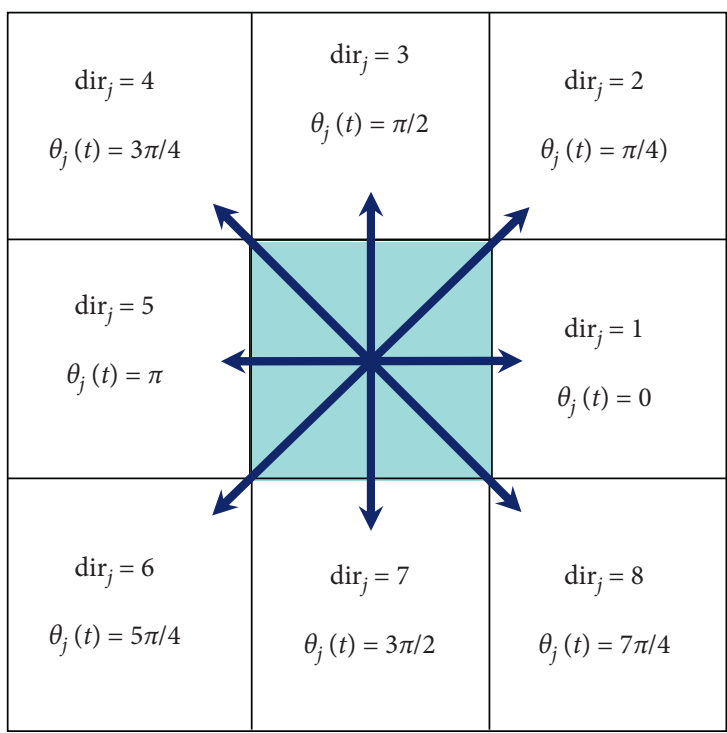

FIGURE 4: Corresponding relationship between forward direction and included angle radian.

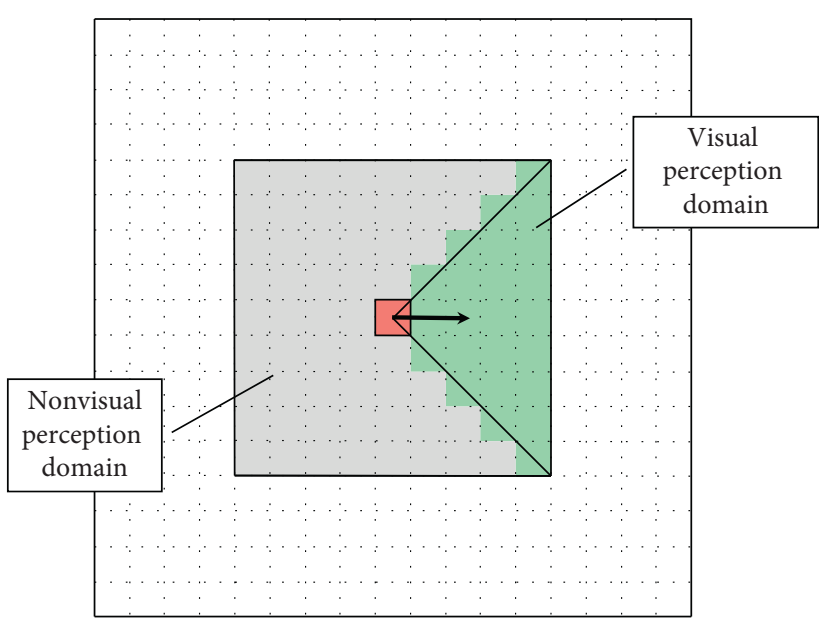

FIGURE 5: Visual perception domain and nonvisual perception domain when $R=2 \mathrm{~m}$.

simulated according to the incomplete rules, such as the location of $n_{2}$ and $n_{3}$. When the evacuation individual is in the other area $A_{3}$, the visual perception domain and nonvisual perception domain are complete, and the simulation calculation can be carried out according to the complete perception domain, such as the location of $n_{4}$ and $n_{5}$.

Nilsson et al. used empirical experiments to analyze other people's influence and behavior choice during indoor evacuation. They proved that the influence of close distance on evacuation individual's emotion and behavior is greater than that of long distance [33]. Fu et al. [29] also believed that the evacuation individuals were more sensitive to the emotional perception of nearby areas during the evacuation process. In this model, the influence of other people's emotion in visual perception domain and nonvisual perception domain on the evacuation individual is different. Combined with the distance factor, this model considers that 


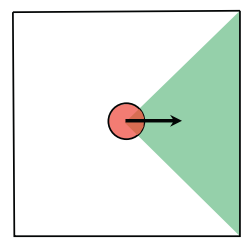

(a)

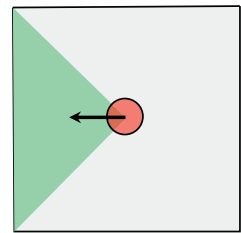

(e)

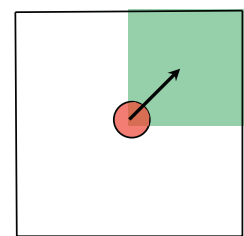

(b)

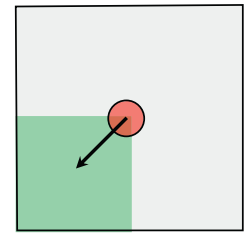

(f)

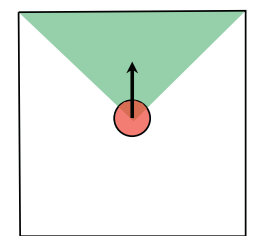

(c)

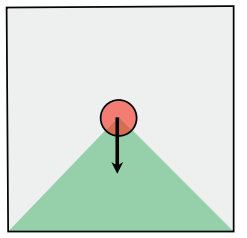

(g)

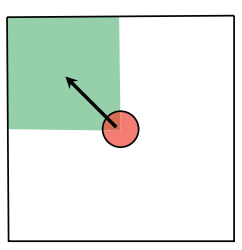

(d)

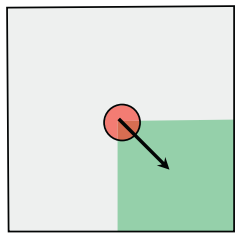

(h)

Figure 6: Possible emotion perception domain of evacuation individual $j$ at time $t$.

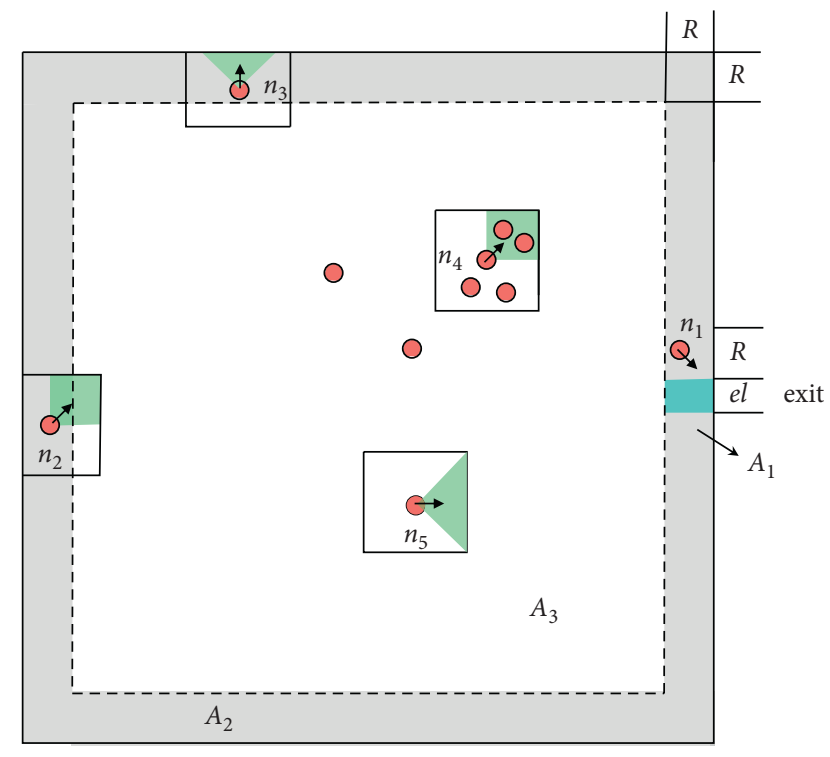

Figure 7: Area division of evacuation space with emotion perception.

the perceived degree of others' emotion in the visual perception domain is greater than that in the visual perception domain and is greater than that in the nonvisual perception domain. Taking Figure 8 as an example, evacuation individual $j$ is more likely to be affected by $n_{6}$, then by $n_{7}$, and finally by $n_{8}$.

In reference [29], the formula of distance as influence weight in emotion gain function is $\left[1-\left(1+\exp \left(-d_{j k}\right)\right)^{-1}\right]$, and $d_{j k}=\sqrt{\left(x_{j}-x_{k}\right)^{2}+\left(y_{j}-y_{k}\right)^{2}}$ is the distance from

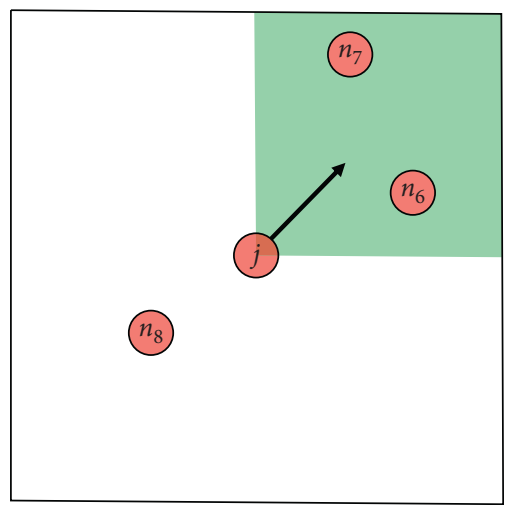

Figure 8: Emotional perception domain of individual $j$.

evacuation individual $j$ to target cell $k$. This formula reflects that evacuation individual $j$ is more sensitive to the emotion of close evacuation individual, and the emotion of others far away from evacuation individual $j$ has less influence. In order to reflect the difference of perception degree in three different situations, this model redefines the distance weight as the following equation:

$$
d d_{k}=\left[1-\frac{1}{1+\exp \left(-d_{j k} \cdot c l\right)}\right] \bullet\left\{\sum\left[1-\frac{1}{1+\exp \left(-d_{j k} \cdot c l\right)}\right]\right\}^{-1}
$$

The average change function of other people's emotion perceived by evacuation individual cell $j$ at time $t$ is shown in the following equation:

$$
\Delta \phi_{j}(t)=\left(g_{1}\right)^{-1} \bullet \sum_{k \in \mathrm{RV}} d d_{k} \times\left[\mathrm{PE}_{k}(t-1)-\mathrm{PE}_{j}(t-1)\right]+\eta \bullet\left(g_{2}\right)^{-1} \sum_{k \in \mathrm{RI}}\left[\mathrm{PE}_{k}(t-1)-\mathrm{PE}_{j}(t-1)\right],
$$

where $g_{1}$ is the number of evacuees in the visual perception domain RV of evacuees cell $j ; g_{2}$ is the number of evacuation individuals in the nonvisual perception domain RI of evacuation individual cell $j ; \eta$ is the degree to which the emotional difference of other people in the nonvisual domain is received by the evacuation individual $j$. Its value is 
not greater than the minimum distance weight in the visual perception domain, that is, $0 \leq \eta \leq \min _{k \in \mathrm{RV}} d d_{j k}$.

The first term of equation (7) represents the average increment of emotional change of evacuation individual $j$ in the visual domain at time $t$. It also shows that the amount of emotional change is related to distance, and the emotional difference in close distance has a great influence on evacuation individual $j$. The latter term indicates that the emotional change of evacuation individual $j$ in the nonvisual domain is the overall weakening due to the absence of specific situation.

In conclusion, the emotional increment function of evacuation individual $j$ at time $t$ is updated as shown in the following equation:

$$
\begin{aligned}
\Delta e_{j}(t)=\Delta f_{j}(v, t)+\Delta \phi_{j}(t)= & \left(1-\frac{v_{j}(t-1)}{v_{j}^{\max }}\right) \bullet \exp \left(-\alpha \bullet\left(\frac{v_{j}(t-1)}{v_{j}^{\max }}\right)\right)+\left(g_{1}\right)^{-1} \bullet \sum_{k \in \mathrm{RV}} d d_{k} \times\left[\mathrm{PE}_{k}(t-1)-\mathrm{PE}_{j}(t-1)\right] \\
& +\eta \bullet\left(g_{2}\right)^{-1} \sum_{k \in \mathrm{RI}}\left[\mathrm{PE}_{k}(t-1)-\mathrm{PE}_{j}(t-1)\right] .
\end{aligned}
$$

The incremental function of emotional intensity indicates that the change of speed will cause the emotional fluctuation of evacuation individuals. When the travel speed is close to the maximum expected speed, the fluctuation of emotion is small. The greater the difference between the travel speed and the maximum expected speed, the greater the emotional fluctuation. The emotions of others around also affect the emotional fluctuation of evacuation individuals [29]. The emotional influence of others in the visual perception domain is greater than that of people in the nonvisual perception domain. That is, the emotional influence of others in the visual perception domain is related to distance [24], and the emotion of evacuation individuals in close distance is easy to be perceived. It can be seen that the constructed incremental function of emotional intensity is more in line with the actual changes of individual emotions.

\subsubsection{Mechanism of Emotional Renewal. The iterative} process of evacuation strategy of evacuation individual $j$ at time $t$ is to judge the state update by emotion update and implement evacuation strategies corresponding to different states. The emotion of evacuation individual $j$ at $t$ time is related to the value of emotion, action strategy, state, and direction at time $t-1$. It is also affected by the emotional intensity of others within the range of emotion perception. The change of emotion will cause the dynamic change of evacuation individual's state and behavior strategy. The specific state update process is shown in Figure 9.

3.3.4. State Update Mechanism. Emotion gathered to a certain extent, resulting in the change of evacuation individual state. According to the SIS model, the evacuation state of the individual is defined as "emotional susceptible" and "emotional infective." The state transition rules follow SIS model. The initial definitions of "emotional susceptible" and "emotional infective" are as follows: when the emotional intensity $\mathrm{PE}_{j}(t)$ of individual $j$ exceeds the threshold $\lambda_{\text {emotion }}$ at time $t$, it is transformed into "emotional infective" by probability $\beta$. When the emotion fluctuation of "emotional infective" is lower than the threshold $\lambda_{\text {emotion }}$, it is transformed into "emotional susceptible" by probability $\gamma$. In the process of evacuation, $S$ is the proportion of "emotional susceptible" and $I$ is the proportion of "emotional infective." Its dynamic differential dynamic equation and system conservation are the same as equation (1).

Different evacuation individuals will adopt different evacuation strategies because of their different states. Literature [34] analyzed the 2010 German "Love Parade" stampede accident video and observed that the crowd presented two different behavioral characteristics, namely, "positive characteristics" and "negative characteristics." People with "positive characteristics" will actively seek exit, accelerate forward, and have obvious herd behavior; people with "negative characteristics" are slow, try to avoid the crowd, and have no obvious herd behavior. Based on this, this model maps pedestrian emotional intensity to pedestrian behavior. During the evacuation, the "emotional susceptible" (S) adopts the "calm" strategy BS, the speed is $v s$, and tends to choose the direction with low density $\rho$; while the "emotional infective" $(I)$ adopts the "impulsive" strategy BI, the speed is $v i$, and tends to choose the direction with high density $\rho$.

$\mathrm{PE}_{j}(t)$ is defined as the emotional intensity of evacuation individual $j$ at time $t$, and the value range is $(0,1]$. That is, the emotion of evacuation individual will not disappear in the whole process of evacuation. According to the different emotional intensity, the evacuation behavior is different. $\lambda_{\text {emotion }}$ is defined as the threshold value of emotional intensity change. It is the critical value from psychology to behavior, and the value range is $(0,1]$. The mapping relationship between emotional intensity and individual behavior type is shown in Table 1.

3.3.5. Transfer Strategy. In this model, cellular automata evacuation model is used. In each time step, the movement direction of the individual who does not leave the evacuation space is determined according to the next time movement probability. Let $P_{j i}(t)$ denote the probability of individual $j$ transferring to cell $C_{i}$ at time $t$. The evacuation individual will choose the neighborhood cell with the maximum transition probability as the target and move at the speed $v_{j}$. According to the theory of cellular automata, the transition probability is related to three basic characteristics such as the 


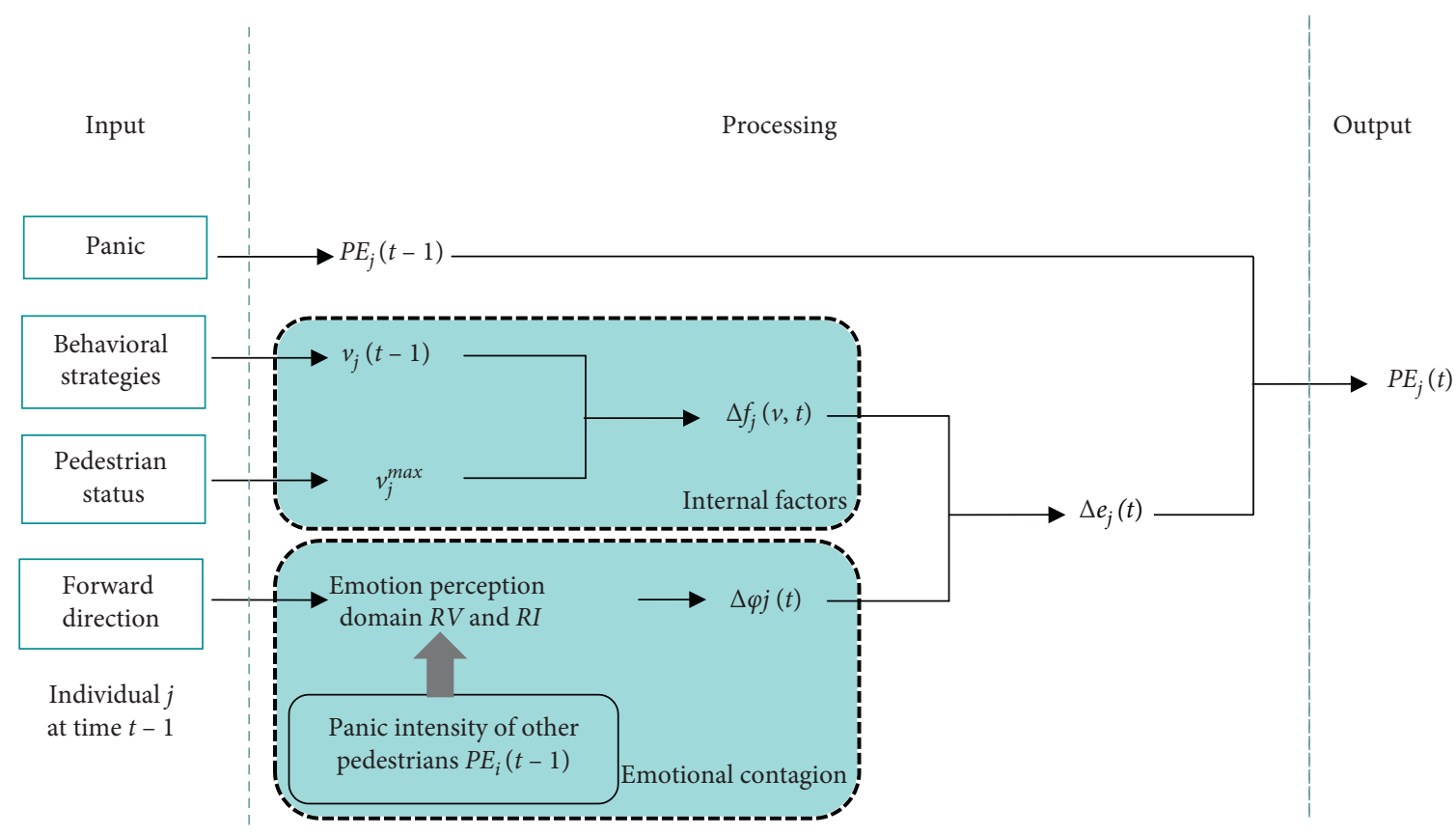

FIgURE 9: Emotional renewal mechanism of evacuation individual $j$ at time $t$.

TABLE 1: Mapping relationship between emotional intensity and behavior types of evacuation individuals.

\begin{tabular}{lcc}
\hline & $\mathrm{PE}_{j}(t) \leq \lambda_{\text {emotion }}$ & $\mathrm{PE}_{j}(t)>\lambda_{\text {emotion }}$ \\
\hline Types of individual evacuation status & $S$ & $I$ \\
Behavior types & "Calm" & "Impulsive" \\
Behavioral strategies & $\mathrm{BS}(v s, \rho)$ & $\mathrm{BI}(v i, \rho)$ \\
\hline
\end{tabular}

distance from neighborhood cell $C_{i}$ to the exit, whether it is an obstacle cell. At the same time, this model considers that the crowd density of the forward direction is also a factor that determines the selection of target cells. Therefore, the cell transition probability is constructed as shown in the following equations:

$$
\begin{aligned}
P_{j i}(t) & =U \bullet \exp \left(k_{s} S_{j i}(t)+k_{\rho} \rho_{j i}(t) \cdot \mathrm{cp}_{j i}(t) \cdot \mathrm{co}_{j i}(t)\right), \\
U & =\left[\sum_{i \text { is all neighbors }} \exp \left(k_{s} S_{j i}(t)+k_{\rho} \rho(t)\right) \cdot \mathrm{cp}_{j i}(t) \cdot \mathrm{co}_{j i}(t)\right]^{-1},
\end{aligned}
$$

where $k_{s}$ is the influence coefficient of distance to the exit; $k_{\rho}$ is the influence coefficient of density.

$\mathrm{cp}_{j i}(t)$ is the state parameter of neighborhood cell $i$ of evacuation individual $j$ at time $t$. If it has been occupied by evacuation individuals, the value is 0 ; otherwise, it is 1 , as shown below:

$$
\mathrm{cp}_{j i}(t)= \begin{cases}0, & C\left(x_{i}, y_{i}\right) \text { isn't empty } \\ 1, & \text { others, }\end{cases}
$$

where $\mathrm{co}_{j i}(t)$ indicates whether the neighborhood cell $i$ of evacuation individual. at time $t$ is an obstacle. If it is an obstacle, the value is 0 ; otherwise, it is 1 , as shown below:

$$
\mathrm{co}_{j i}(t)= \begin{cases}0, & C\left(x_{i}, y_{i}\right) \text { is barrier, } \\ 1, & \text { others, }\end{cases}
$$

where $\rho_{j i}(t)$ is the density around the neighborhood cell $i$ of the evacuation individual $j$ at time $t$. At present, all other evacuation individuals in the visible area of evacuation individuals will affect their choice. This model gives the density influence function of different types of groups combined with the decision types. Therefore, the transfer probability function of different types of crowd is different.

(1) "Emotional susceptible" $S$ adopts "calm" strategy BS. The maximum expected speed is $v_{j}^{\max }=1 \mathrm{~m} / \mathrm{s}$. That is, when each step is updated, it moves 1 step. The target cell of "calm type" evacuation is 8 cells around, as shown in Figure 10.

At the same time, the density range of "calm type" evacuation individuals is the close density around the neighborhood cell $i$. As shown in Figure 10, the red cell is 


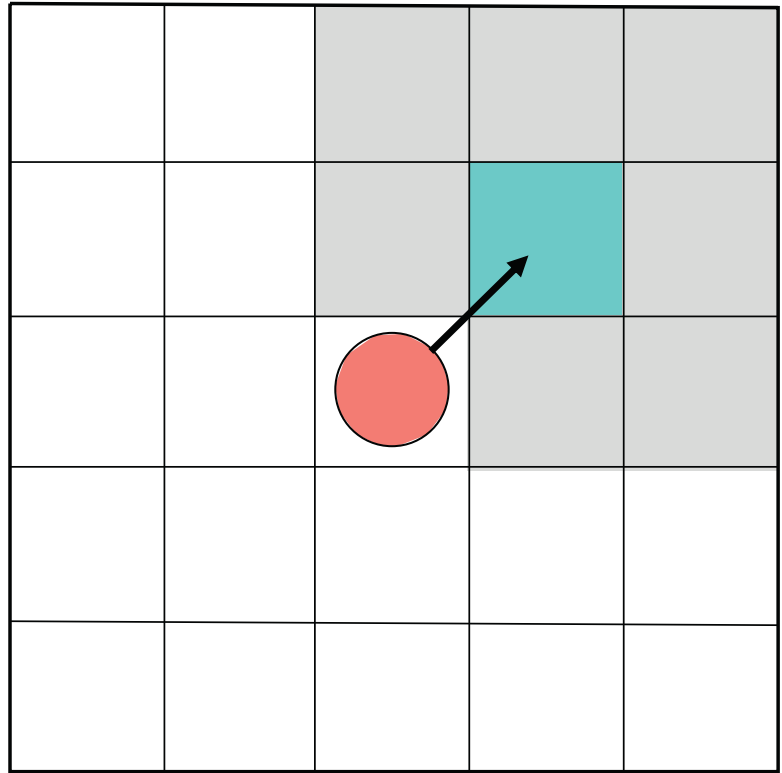

Figure 10: Density range observed for "calm."

evacuation individual $j$, and the blue cell is neighborhood cell $i$. When analyzing the density of $i$, evacuation individual $j$ considers the density of the gray cell. Because the "calm" evacuation individuals described in this model choose the direction with low crowd density, the transfer probability formula of this kind of crowd is as follows:

$$
\begin{aligned}
P_{j i}(t)= & U \bullet \exp \left(k_{s} S_{j i}(t)+k_{\rho}\left(1-\rho_{j i}(t)\right) \cdot c p_{j i}(t) \cdot c o_{j i}(t)\right)\left[\sum_{\text {iisallneighbors }} \exp \left(k_{s} S_{j i}(t)+k_{\rho}(1-\rho(t))\right) \cdot c p_{j i}(t) \cdot c o_{j i}(t)\right]^{-1} \\
& \bullet \exp \left(k_{s} S_{j i}(t)+k_{\rho}\left(1-\rho_{j i}(t)\right) \cdot c p_{j i}(t) \cdot c o_{j i}(t)\right) .
\end{aligned}
$$

(2) "Emotional infective" I adopts "impulsive" strategy BI. The velocity is $v i$. They tend to choose the direction with high crowd density $\rho$. The maximum expected speed is $v_{j}^{\max }=2 \mathrm{~m} / \mathrm{s}$. When each step is updated, it moves 2 steps. Then, the target cell of "impulsive" evacuation is two-step cell in each direction. Suppose the group of target cells is set $G_{1}$, as shown in the blue cells in Figure 11(a). At this time, the moving speed is $v i=v_{j}^{\max }$. If all the preferred eight cells are occupied, the target cell with the largest transition probability of eight cells in neighborhood is selected. Suppose the neighborhood cell is set $G_{2}$, as shown in the blue cells in Figure 11(b). At this time, the moving speed is $v i=1 \mathrm{~m} / \mathrm{s}$.

The radius of density range of "impulsive" evacuation individuals is two steps, as shown in Figure 12.

Because the "impulsive" evacuation individuals described in this model choose the direction with high crowd density, the calculation method of the transition probability of this kind of crowd is as follows:

Calculate $P_{j i}(t)$ according to equation (9). If

$$
\sum_{i \in G_{1}} P_{j i}(t)=0,
$$

then calculate $P_{j i}(t)$.

$$
i \in G_{2}
$$

\section{Simulation Experiment}

4.1. Simulation Process. According to the emotional intensity of individual evacuation, the individual evacuation is divided into two groups: "emotional susceptible" and "emotional infective." The "calm" strategy and "impulsive" strategy are adopted in evacuation. The emotional intensity of individual evacuation is spread by others and fluctuates, and the evacuation strategy of each individual evacuation is not single. Moreover, due to the influence of dynamic changes, the emotion of evacuation individuals affects their mobile behavior [34]. Considering that only when emotion accumulates to the time step of cell movement can pedestrian movement be driven, the improved model adopts parallel update mechanism with a unified time step $\Delta t$. In each time step $\Delta t$, the emotion is updated firstly to judge the change of the emotional state. Then, the behavioral decisionmaking is updated. The simulation process of this model is as follows:

(1) Initialize the number of people and the layout of evacuation space;

(2) Calculate the SFF of each cell according to equation (2); 


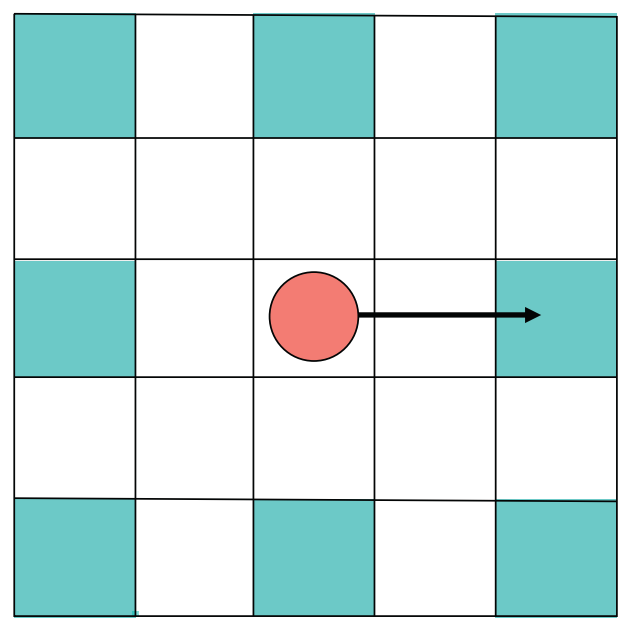

(a)

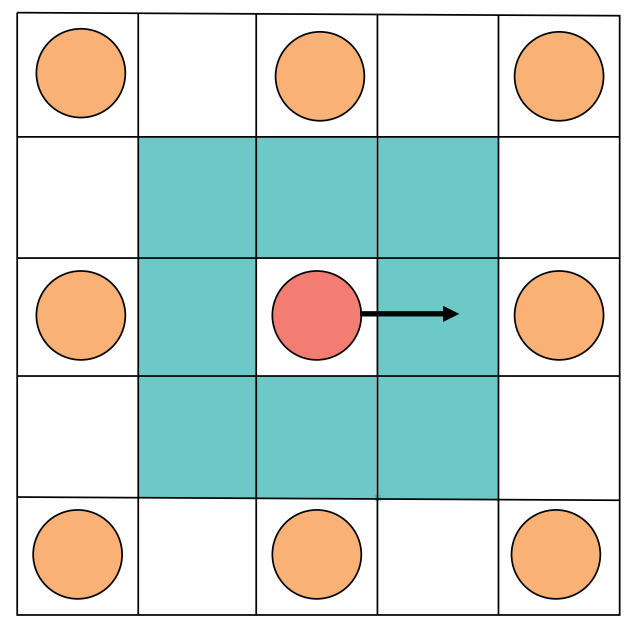

(b)

Figure 11: Target cell of "impulsive."

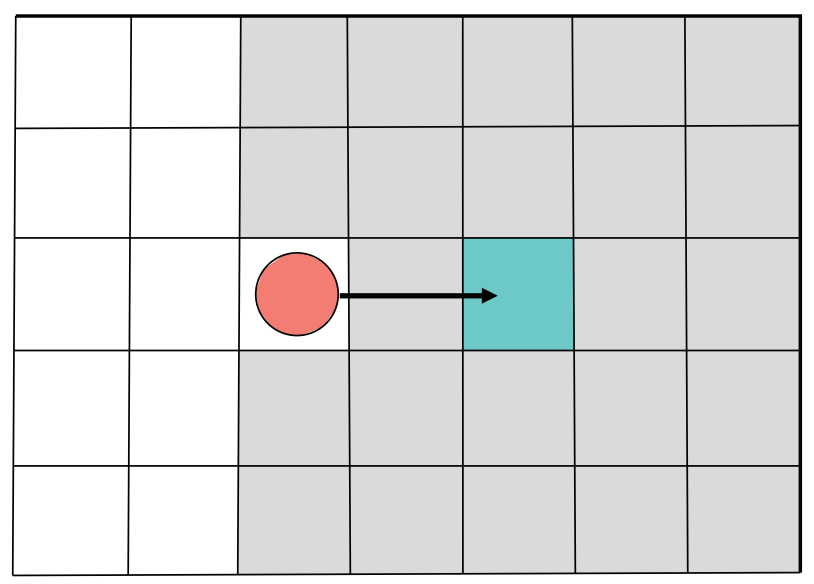

FIGURE 12: Density range observed for "impulsive."

(3) Distribute the crowd in the evacuation space randomly and initialize the emotional intensity $\mathrm{PE}_{j}(0)$ of evacuation individuals. When the emotional intensity of individual is lower than the threshold $\lambda_{\text {emotion, }}$ record the location and emotional intensity of individual in the initial set $S$. Otherwise, record them in the initial set $I$;

(4) Update individual emotion: calculate the emotional increment according to equations (3)-(8) and update the emotional intensity;

(5) Update individual status: if the emotional intensity of "emotional susceptible" $S$ is higher than the threshold $\lambda_{\text {emotion }}$, it will turn into "emotional infective" $I$ with probability $\beta$; if the emotional intensity of "emotional infective" $I$ is lower than the threshold $\lambda_{\text {emotion }}$, it will turn into "emotional susceptible" $S$ with probability $\gamma$. Realize the dynamic change of evacuation crowd state, as shown in Figure 13;

(6) Calculate the individual transfer strategy according to equations (9)-(14) and select the target cell;
(7) Solve conflicts: when multiple evacuees choose the same target unit, there will be conflicts. In this case, an evacuation individual is randomly selected to move. For simplicity, it is assumed that the probability of each individual being selected is equal [31];

(8) When all the evacuees in the space leave, the simulation ends; otherwise, jump to (4);

(9) Every time the evacuation individual moves, pedometer +1 , until the end of the simulation.

\subsection{Simulation Verification Analysis}

4.2.1. Experiment 1. This experiment analyzes the influence of perception domain on the total evacuation time. In the improved model, the emotion perception domain of the evacuation individual is a circle with a radius $R$. Set a single exit room as the simulation scene. The size is $12 \mathrm{~m} \times 12 \mathrm{~m}$. The exit width is $e l_{1}=3 \mathrm{cl}$. The size of each cell is $\mathrm{cl} \times \mathrm{cl}$, $\mathrm{cl}=0.4 \mathrm{~m}$. The initial number of people is $N=200$, which is randomly distributed in the space and given the initial emotion value. In order to analyze the influence of perception domain radius $R$, set $n n=R / \mathrm{cl}(\mathrm{cl}=0.4 \mathrm{~m})$. Then, $n n=1,2,3,4,5$. Emotional intensity threshold $\lambda_{\text {emotion, }}$, proportion of emotional infected people $I$, emotional infection coefficient $\beta$, and emotional calm coefficient $\gamma$ and other parameters all affect the overall evacuation time of the system. Therefore, three different scenarios with different parameters are set for simulation experiments. Scenario 1: $\beta=0.33, \gamma=0.67$; scenario $2: \beta=0.5, \gamma=0.5$; scenario 3 : $\beta=0.67, \gamma=0.33$. Next, we analyze the impact of different perception domain radius $n n$ on the total evacuation time under three scenarios, when the emotional threshold $\lambda_{\text {emotion }}=0.2,0.4,0.6,0.8$. Each experiment runs 100 times, and the average of the total evacuation time is taken. The simulation results are shown in Figures 14-16. It can be seen 


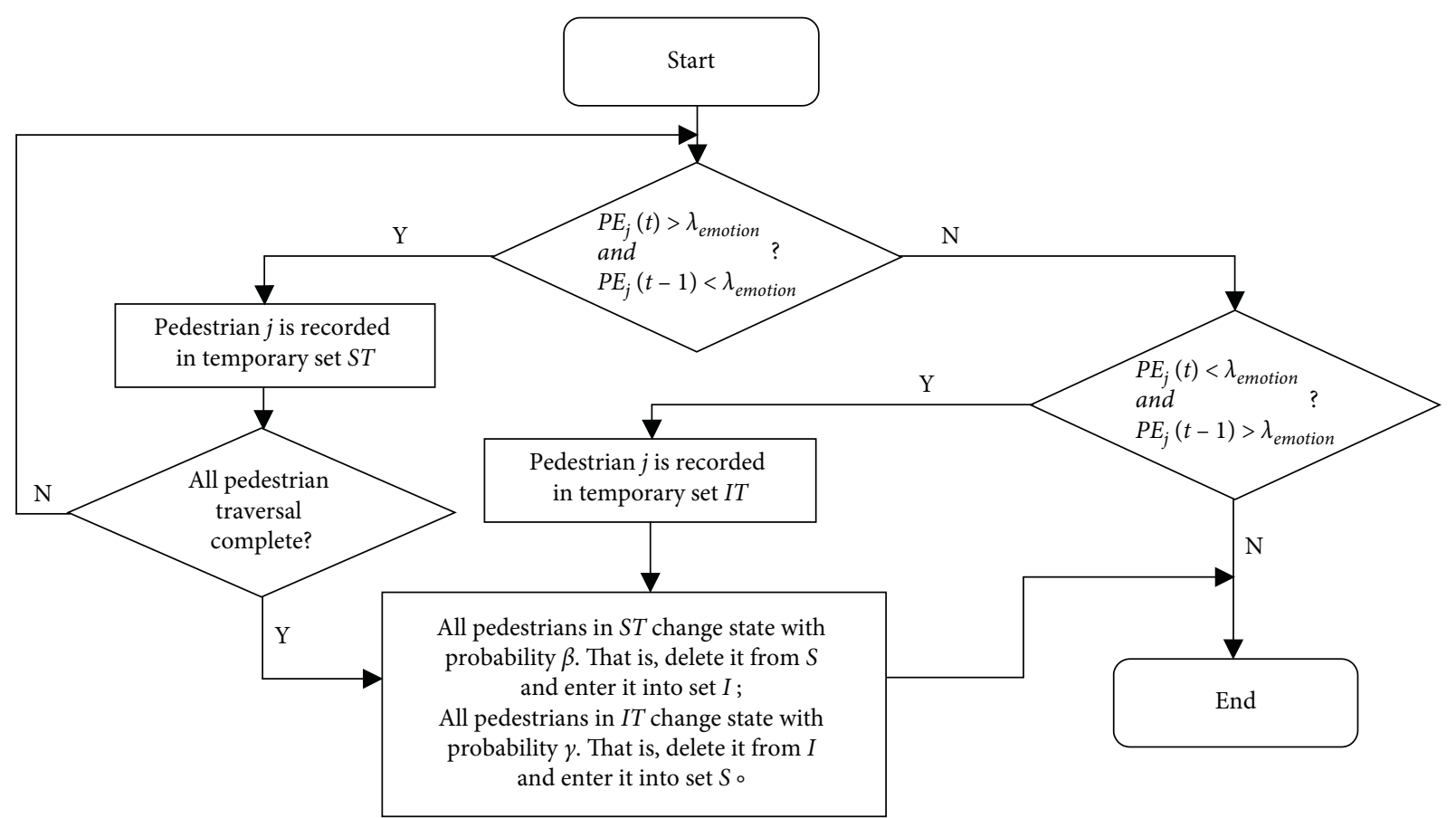

FIGURE 13: Update process of evacuation individual state in time $t$.

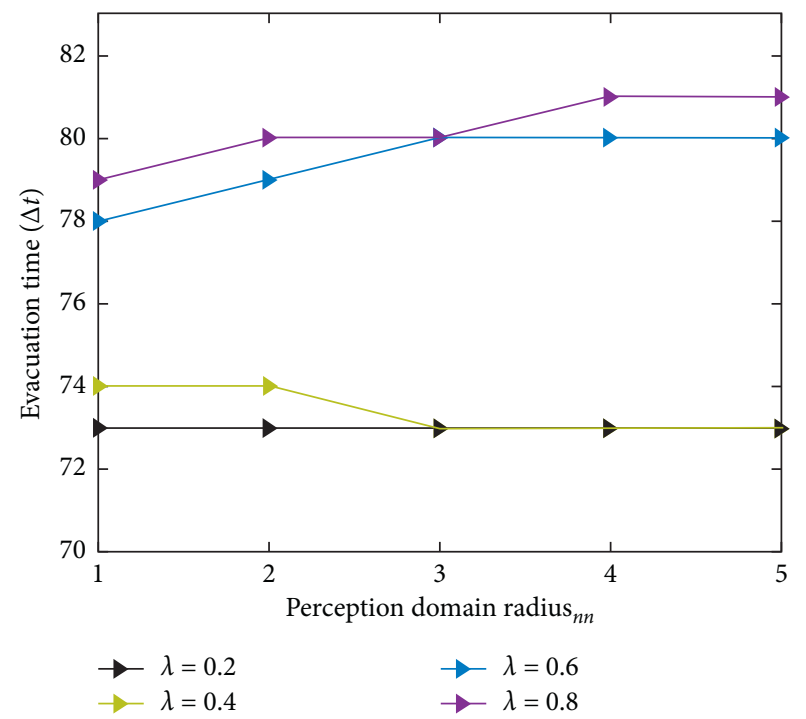

FIgURE 14: Total evacuation time with different perception domain radius in scenario $1(\beta=0.33, \gamma=0.67)$.

from the figure that, when $\lambda_{\text {emotion }}=0.2$, there is no difference in the total evacuation time between scenario 1 and scenario 3, while the total evacuation time of the perceived radius $n n=4.5$ is smaller at scenario 2 ; When $\lambda_{\text {emotion }}=0.4$, the total evacuation time of the perceived radius $n n=4.5$ is less than $n n=1,2,3$ in the three scenarios. When $\lambda_{\text {emotion }}=$ 0.6 and $\lambda_{\text {emotion }}=0.8$, the three scenarios show that the minimum total evacuation time appears when $n n=1$, which generally shows that the larger the perception domain radius is, the larger the total evacuation time is. Therefore, when $\lambda_{\text {emotion }}<0.5$, the larger the perceived radius $n n$ is, the faster the total evacuation time is; when $\lambda_{\text {emotion }}>0.5$, the larger the perceived radius $n n$ is, the longer the total evacuation time is. The values of $\beta$ and $\gamma$ have no decisive influence on this conclusion. In order to verify this conclusion, we carried out simulation experiments on the same three scenarios with the initial numbers of people $N=300$ and $N=400$, and the results are consistent.

4.2.2. Experiment 2. The perceived domain radius of individual evacuation has a direct impact on the emotion of individual evacuation. We carried out simulation experiments on the mean system emotions in scenario 1, scenario 2 , and scenario 3 under different emotion thresholds. The experimental results are shown in Figure 17. In the figure, the horizontal axis is the perception domain radius $n n=1,2,3,4,5$. The vertical axis is the mean emotional value of the whole system, and the formula is as follows: $\operatorname{APE}(t)=\sum_{j=1}^{N(t)} \mathrm{PE}_{j} / N(t)$, that is, the cumulative sum of the mean system emotion at each time divided by the total evacuation time. The value of this parameter reflects the emotional level of the individual in the process of evacuation. The initial position and initial emotional value of the experiment were the same as that of experiment 1 . The results show that, when $\lambda_{\text {emotion }}=0.2$ and $\lambda_{\text {emotion }}=0.4$, with the increase of perception domain radius, the mean system emotion increases; when $\lambda_{\text {emotion }}=0.6$ and $\lambda_{\text {emotion }}=0.8$, with the increase of perception domain radius, the mean system emotion decreases. The conclusion of this experiment is that $\lambda_{\text {emotion }}=0.5$ can be regarded as a cut-off point of emotional value. The initial people numbers of $N=300$ and $N=400$ are simulated, and the conclusion is consistent. 


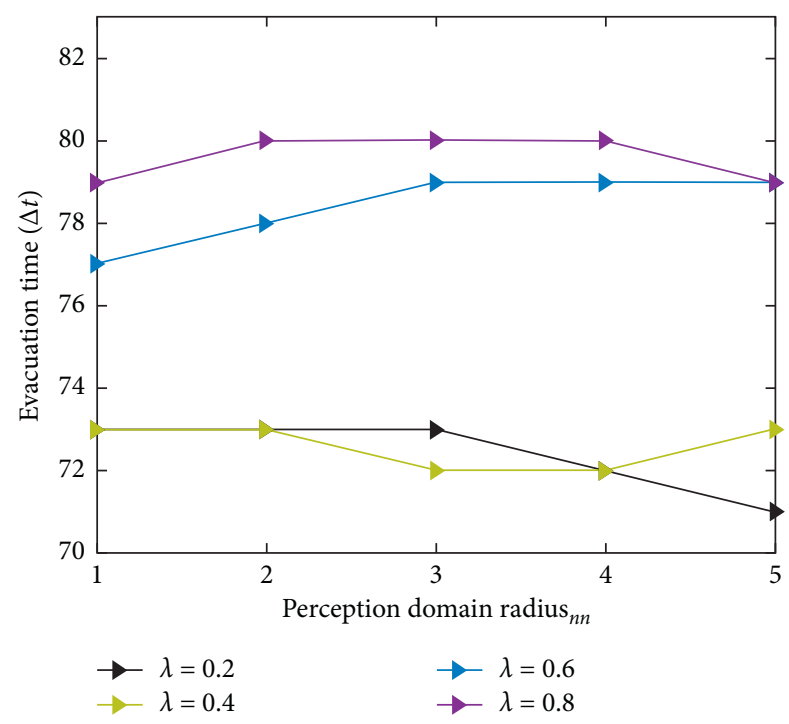

FIgURE 15: Total evacuation time with different perception domain radius in scenario $2(\beta=0.5, \gamma=0.5)$.

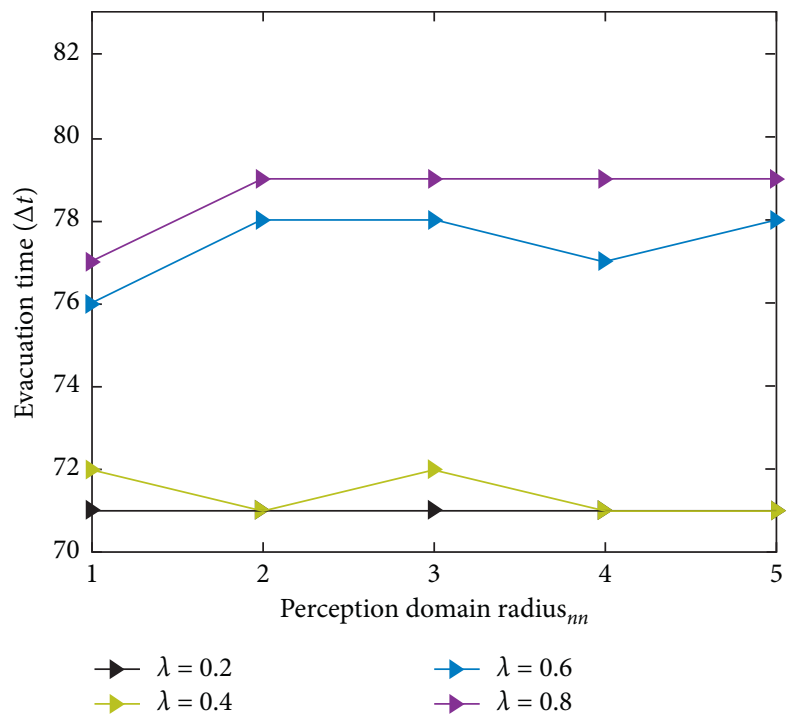

Figure 16: Total evacuation time with different perception domain radius in scenario $3(\beta=0.67, \gamma=0.33)$.

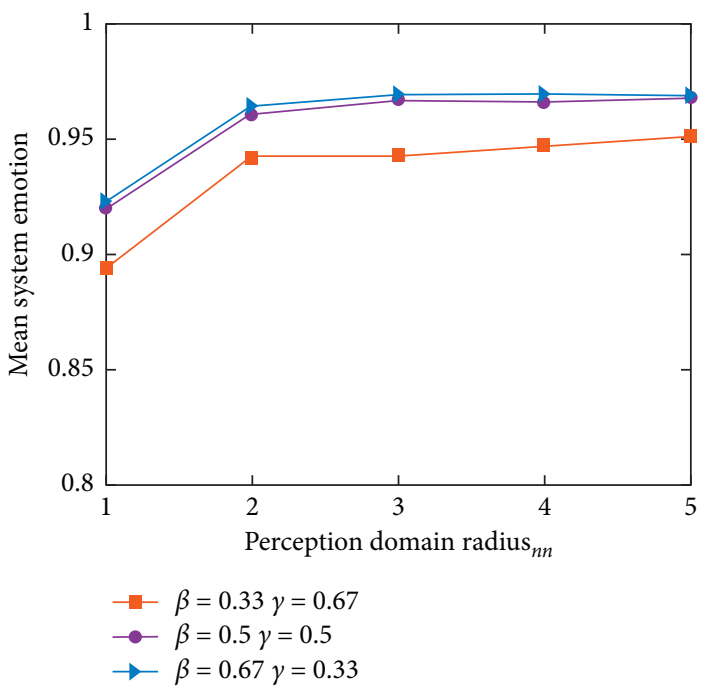

(a)

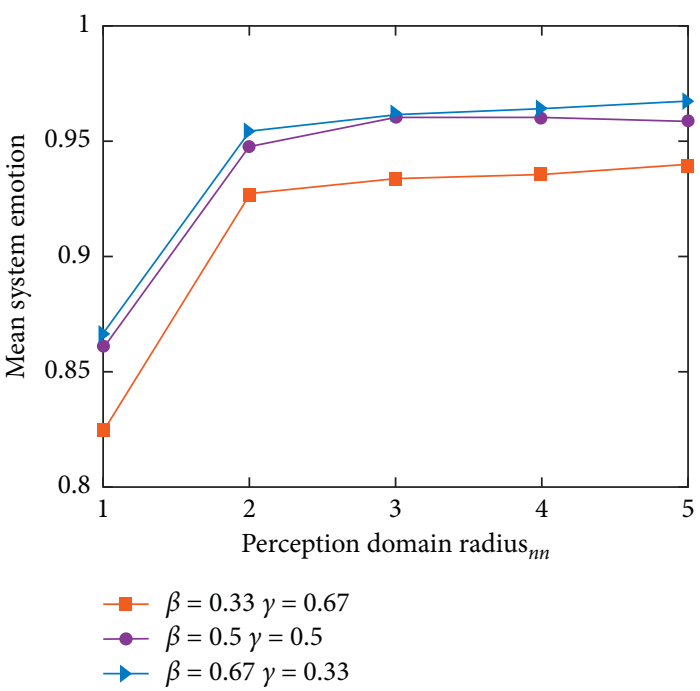

(b)

Figure 17: Continued. 


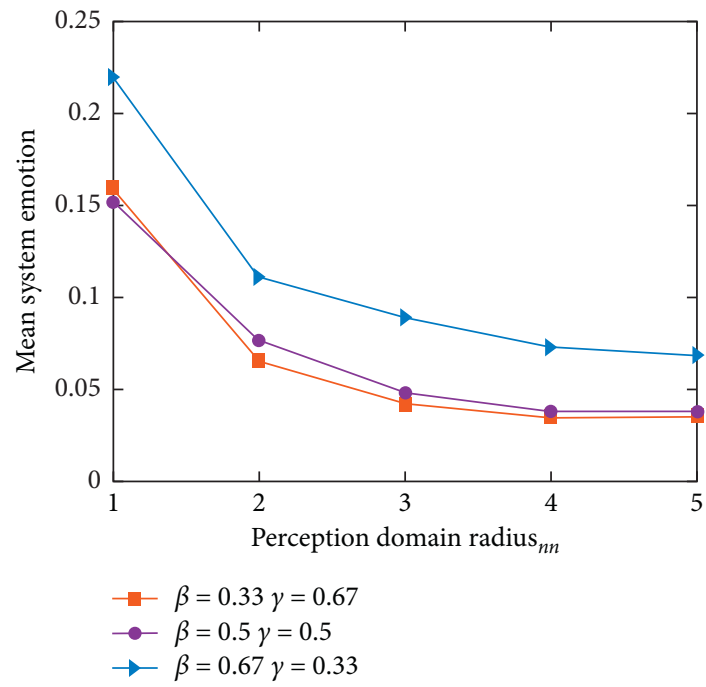

(c)

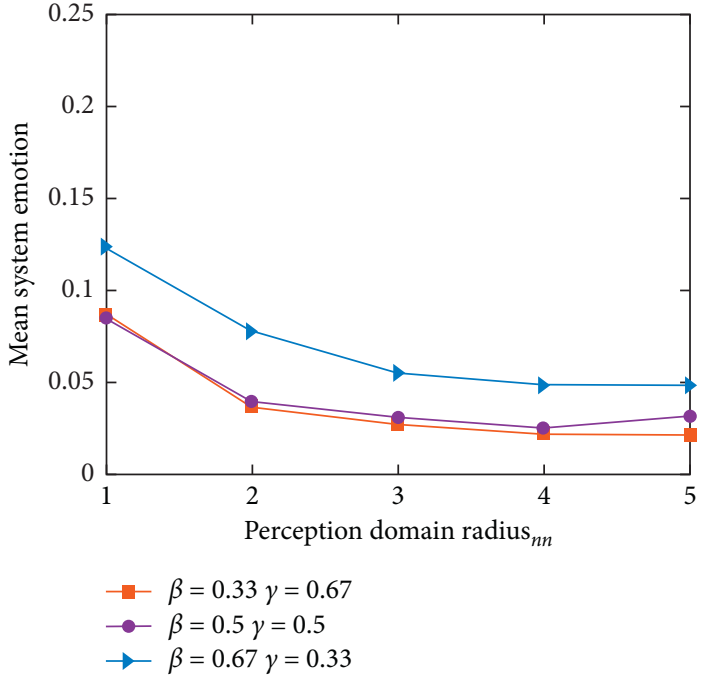

(d)

Figure 17: Mean system emotion value under different initial conditions and different perception domain radius. (a) $\lambda_{\text {emotion }}=0.2$. (b) $\lambda_{\text {emotion }}=0.4$. (c) $\lambda_{\text {emotion }}=0.6$. (d) $\lambda_{\text {emotion }}=0.8$.

4.2.3. Experiment 3. The change of emotional value will lead to the change of evacuation individual state. This experiment analyzes the influence of different perceived domain radius of evacuation individuals on the state of evacuation individuals. According to the previous two experiments, $\lambda_{\text {emotion }}=0.5$ is the inflection point of the change of evacuation time and mean system emotional value. In order to observe the detailed changes of pedestrian status more clearly, $\lambda_{\text {emotion }}=0.4, \lambda_{\text {emotion }}=0.5$, and $\lambda_{\text {emotion }}=0.6$ are taken in this experiment respectively, to analyze the change of the proportion of state $I$ in the system at each time. The experiment adopts the same scene as the previous experiment. The initial position and initial emotional value are the same. The initial number of people are $N=200$ and $N=300$ respectively. Take $\beta=0.5$ and $\gamma=0.5$. The experimental results are shown in Figures 18 and 19.

It can be seen from the figure that no matter $N=200$ or $N=300$, the system will be transformed into group $I$ eventually with the evacuation time when $\lambda_{\text {emotion }}=0.4$. When the perception domain radius $n n=1$, the change speed is slower, and when $n n=2,3,4,5$, the change speed is faster. The proportion curve of state $I$ of perception domain radius $n n=1$ at $\lambda_{\text {emotion }}=0.5$ is different from other situations significantly. When $N=200$, the proportion of $I$ increases first and then decreases rapidly. In the last stage of evacuation, group $I$ leaves first, and the last one is individual $S$. So, at the last moment, $S=1$. When $N=300$, the number of group $I$ decreased slowly. This shows that for $n n=1$, it is more beneficial to transform into group $I$ when the number of people is small. But state group $I$ and state group $S$ always coexist in the system under the critical threshold no matter what kind of initial number. Under two initial numbers, when the perception domain radius $n n=2,3,4,5$, the evacuation individual status in the system is ultimately group $I$. From the curve change, when $n n=2$, the change speed of $I$ state group is slightly slower than that of $n n=3,4,5$. When $\lambda_{\text {emotion }}=0.6$, whether the initial number is $N=200$ or $N=300$, with the change of evacuation time, the system will be all group $S$. It can be seen that the state change speed of perception radius $n n=1$ is the slowest, followed by $n n=2$. When $N=200$, the change speed of group $I$ of $n n=3$ is slightly slower than that of $n n=4$ and $n n=5$, while when $N=300$; the difference of curves of $n n=$ $3,4,5$ is small. Therefore, when $\lambda_{\text {emotion }} \leq 0.5$, the larger the perceived domain radius of the evacuation individual is, the more favorable it is for the evacuation individual to change into state $I$. When $\lambda_{\text {emotion }}>0.5$, the larger the perceived domain radius of the evacuation individual, the easier the evacuation individual is to change to state $S$.

4.2.4. Experiment 4. The improved model and CA model are compared in the same scenario to verify the effectiveness of the model. The basic scene of reference [24] is used as the simulation scene. The unit cell is $\mathrm{cl} \times \mathrm{cl}, \mathrm{cl}=0.4 \mathrm{~m}$. The single exit room size is $18 \mathrm{cl} \times 14 \mathrm{cl}$, and the exit width is $e l_{1}=2 \mathrm{cl}$. When the initial state $t=0,150$ individuals were randomly distributed in the space, each occupying a cell. Moore's neighbor rule is used when evacuation individuals move. Other parameters of this model are set as $\lambda_{\text {emotion }}=0.6$, $\xi=0, \beta=1$, and $\gamma=1$. CA model does not consider emotional contagion, only according to the SFF rule evacuation model. After the evacuation starts, the evacuation individuals move to the exit according to different model moving rules. Each time step is 0.4 s. Figures 20 and 21 record the density maps of the two models at different time steps, respectively. The cell density function is shown in equation (15). It follows Moore's neighbor rule, so num $=8$ in the formula. $\mathrm{cp}_{j i}(t)$ denotes whether there are people in the neighbor cell of cell $j$ at time $t$. If $\mathrm{cp}_{j i}(t)=1$, the neighbor cell has people; otherwise, the neighbor cell has no people. 


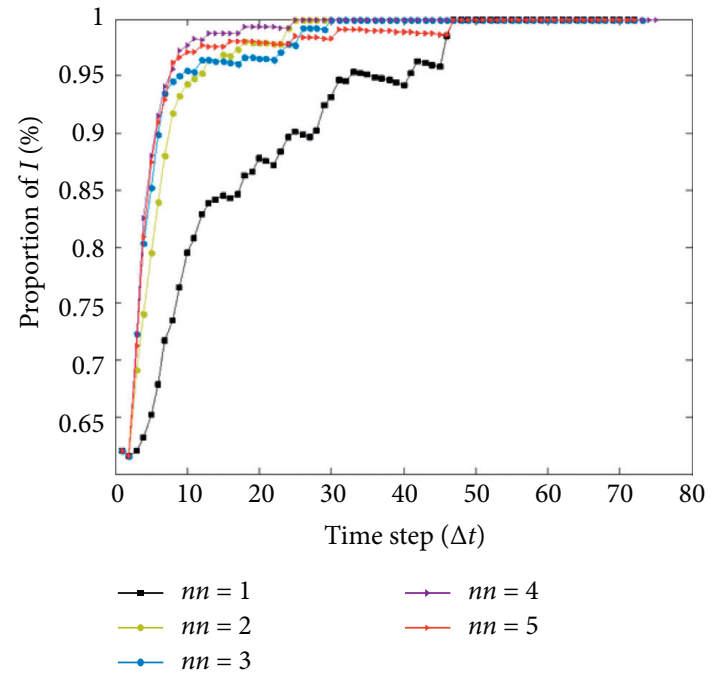

(a)
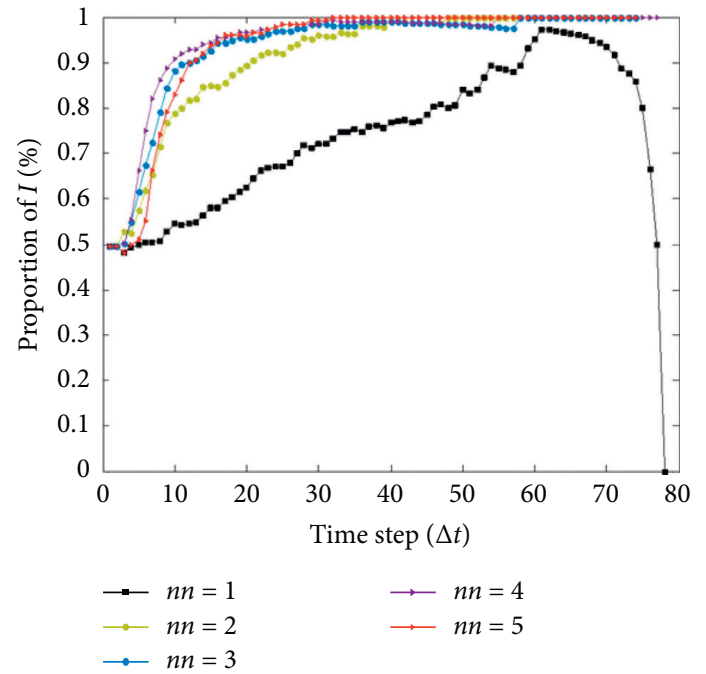

(b)

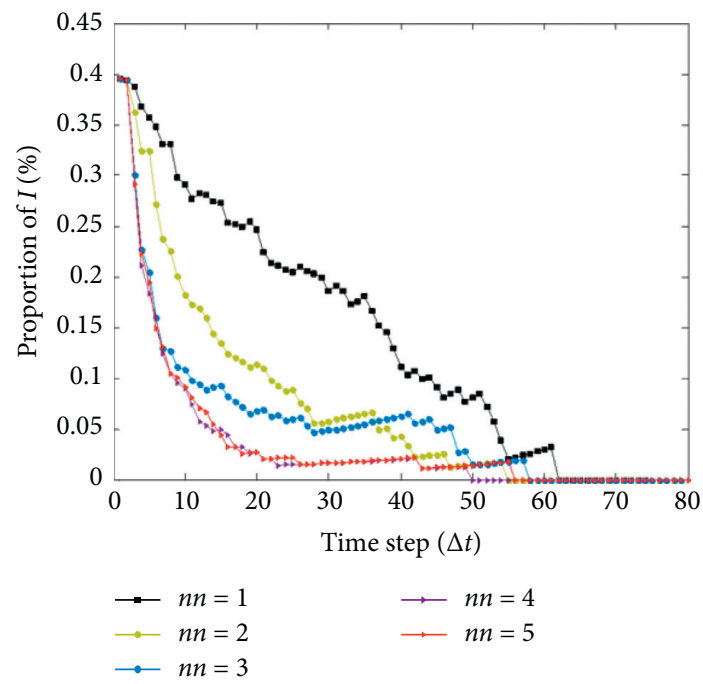

(c)

Figure 18: Proportion of state group $I$ under different perception radius when $N=200$. (a) $\lambda_{\text {emotion }}=0.4$. (b) $\lambda_{\text {emotion }}=0.5$. (c) $\lambda_{\text {emotion }}=0.6$.

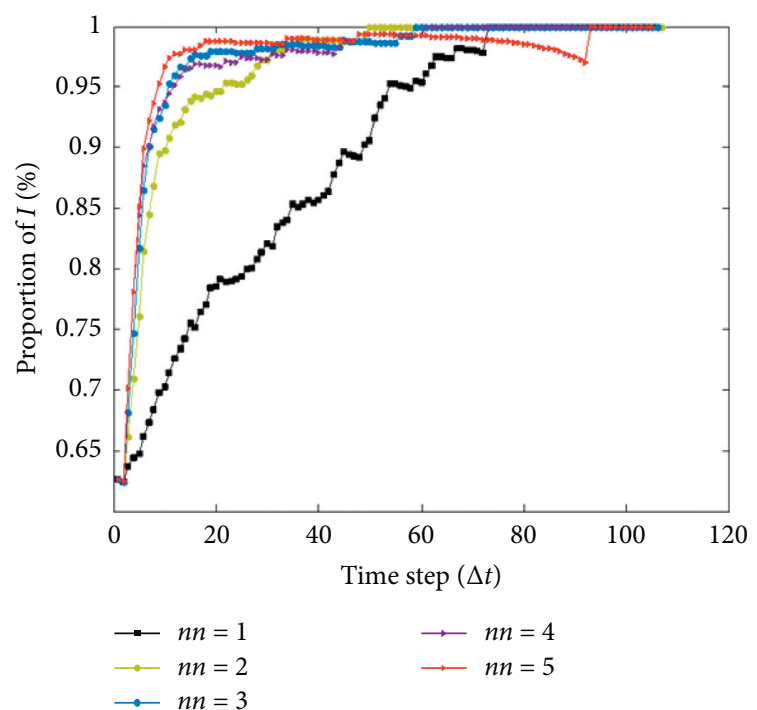

(a)

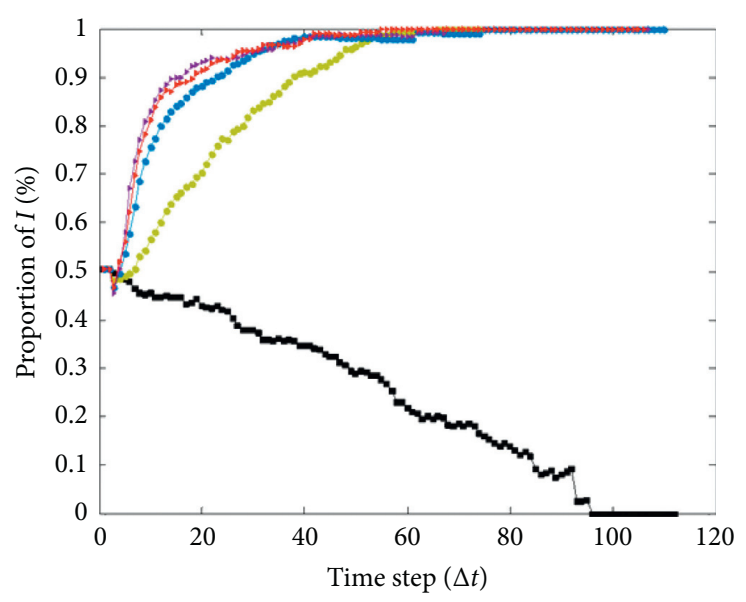

$$
\begin{aligned}
& \because n n=1 \quad \longrightarrow n n=4 \\
& \because n n=2 \quad \longrightarrow n n=5
\end{aligned}
$$$$
\because n n=3
$$

(b)

Figure 19: Continued. 


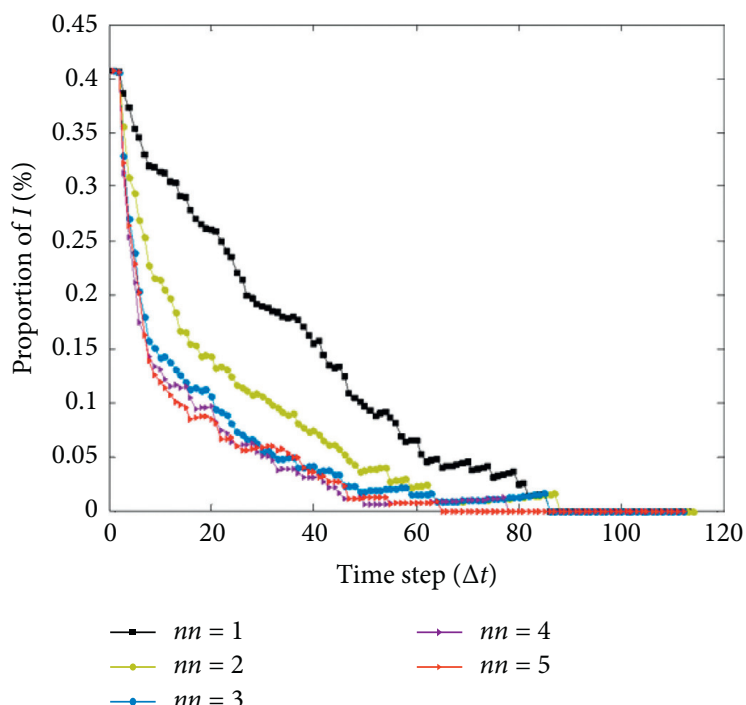

(c)

Figure 19: Proportion of state group $I$ under different perception radius when $N=300$. (a) $\lambda_{\text {emotion }}=0.4$. (b) $\lambda_{\text {emotion }}=0.5$. (c) $\lambda_{\text {emotion }}=0.6$.

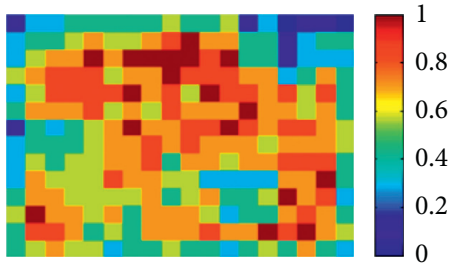

(a)

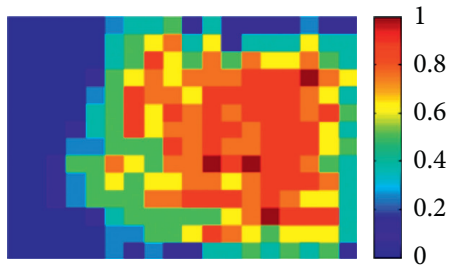

(b)

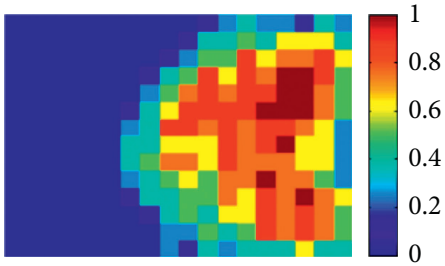

(c)

Figure 20: Diagram of personnel density of CA model at different times (unit: $\Delta t$ ). (a) $T=0$. (b) $T=40$. (c) $T=80$.

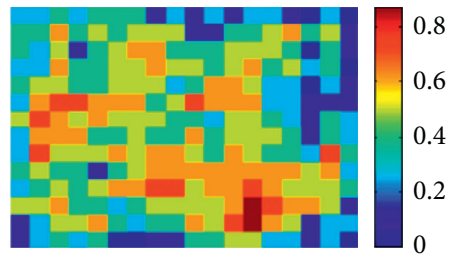

(a)

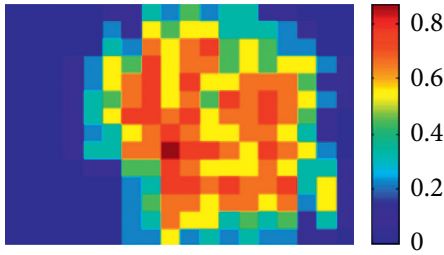

(b)

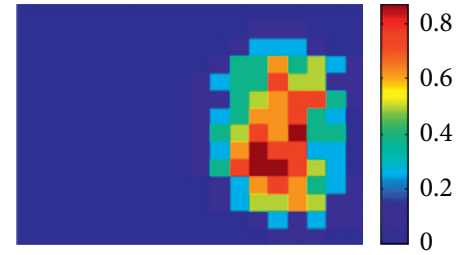

(c)

Figure 21: Diagram of personnel density of the improved model at different times (unit: $\Delta t$ ). (a) $T=0$. (b) $T=40$. (c) $T=80$.

$$
\rho_{j}=\frac{\sum_{i=1}^{\mathrm{num}} \mathrm{cp}_{j i}(t)}{\operatorname{num}} .
$$

As can be seen from the figure, the personnel movement trend of the improved model is consistent with the CA model. The pedestrian flow at the exit is arched, which is in line with the characteristics of individual movement flow. In the same time step, the evacuation individuals move faster in the improved model. This is because the emotional contagion of evacuation individuals leads to the dynamic movement speed, which reduces the overall evacuation time.
4.2.5. Experiment 5. In order to further verify that the improved model can reflect the movement characteristics of individual evacuation, the scene of reference [24] is used to carry out the experiment. $N$ people to be evacuated are distributed in a single exit room with the size of $12 \mathrm{~m} \times 12 \mathrm{~m}$ randomly. Each cell is $\mathrm{cl} \times \mathrm{cl}, \mathrm{cl}=0.4 \mathrm{~m}$. That is, there are 900 cells in a single exit room. Suppose the total number of people is $N=100,200,300,400,500,600,700,800$, and the exit width is $2,3,4,5,6,7$, and 8 respectively. Comparing the total evacuation time of the improved model with that of the CA model, the simulation results are shown in Figures 22(a) and 22(b). 


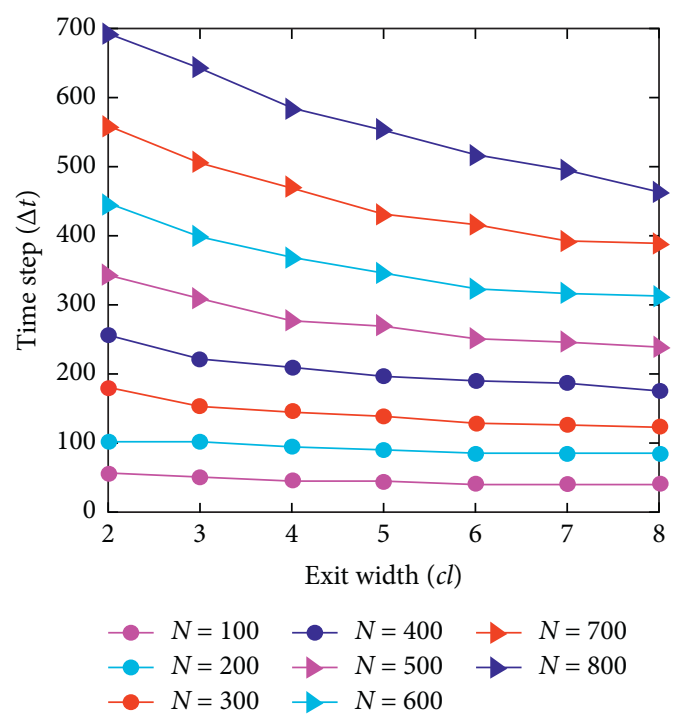

(a)

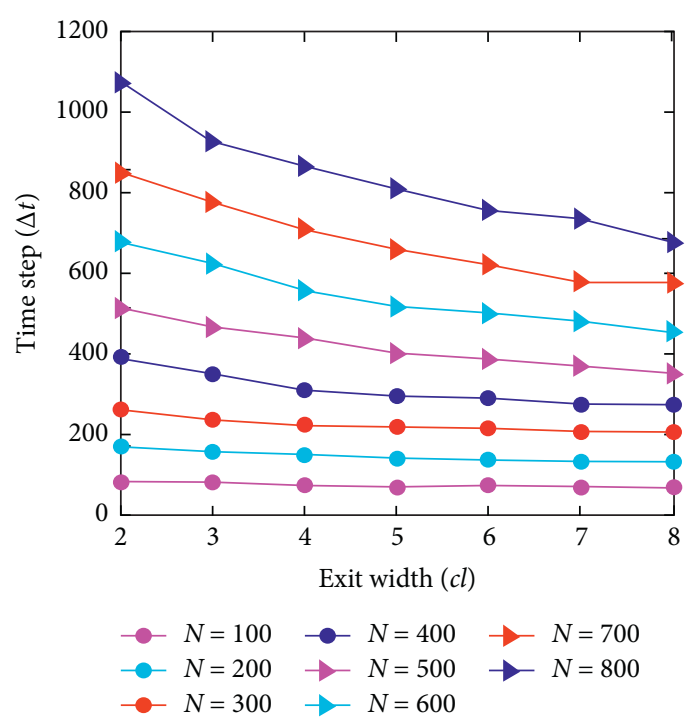

(b)

Figure 22: Total evacuation time steps of the improved model and CA model with different number of people. (a) Improved model. (b) CA model.

It can be seen from the figure that the trend presented by the two models is consistent. The wider the exit width is, the smaller the evacuation time is. The evacuation time of the improved model is shorter than that of the CA model under the same number of people and the same exit width. The reason is that the improved model takes into account the emotional contagion of individual evacuation, and different emotional groups adopt different evacuation strategies.

\section{Conclusion}

In this paper, the SIS model of infectious diseases is combined with cellular automata, and a crowd evacuation model considering emotional contagion is proposed. In the improved model, the dynamic movement rules of evacuation individuals are constructed. The action strategy, state transition rules, emotion update function, and linkage relationship among them are refined.

Three simulation experiments were carried out to study the influence of perception domain radius on the total evacuation time, the mean system emotion, and the proportion of state group I under different emotional threshold, infection coefficient, and calm coefficient. The results show that when the system emotion threshold $\lambda_{\text {emotion }} \leq 0.5$, the larger the perceived domain radius, the higher the mean system emotion value, the easier the evacuation individual is to change to state $I$ and leave the site quickly. When $\lambda_{\text {emotion }}>0.5$, the larger the perceived domain radius, the lower the mean system emotion value, and the easier the evacuation individual is to change to state $S$, resulting in a longer evacuation time. The conclusion can provide the following evacuation guidance for evacuation individuals. When $\lambda_{\text {emotion }} \leq 0.5$, the field of vision of the evacuation individual can be enlarged and the observation area is far away. This is conducive to judging the critical situation, forming a convergent emotion, and then make positive action strategies. When $\lambda_{\text {emotion }}>0.5$, the evacuation individual pays attention to the emotion or behavior strategy of others nearby, which is more conducive to change personal emotion and state. If the observation scope is too large, it may lead to some misjudgments and adopt a calm strategy, resulting in prolonged evacuation time.

Two simulation experiments are compared with the CA model. The results show that the improved model can effectively simulate the process of individual evacuation, showing the characteristics of crowd conformity and exit arch. In addition, the comparative analysis also shows that emotional contagion has an impact on evacuation time. Because different emotional individuals choose different evacuation strategies, evacuation individuals move faster than the traditional CA model.

In summary, this model can not only realize the dynamic characteristics of individual state and emotion in the macroevacuation model but also analyze the microcharacteristics of individual state change and emotion fluctuation. This conclusion provides a basis for managers to grasp the characteristics of emotional contagion among people and put forward effective control strategies. Of course, the content of this chapter needs to be improved, and the simulation results need to be supported by empirical data. In the future, empirical experiments will be conducted to verify the significance of the conclusions in this chapter.

\section{Data Availability}

The data used to support the findings of this study are included within the article.

\section{Conflicts of Interest}

The authors declare that they have no conflicts of interest. 


\section{Acknowledgments}

This work was supported in part by the Natural Science Foundation of Liaoning Province under Grant 2019-ZD0558.

\section{References}

[1] H. Kim and S. Han, "Crowd evacuation simulation using active route choice model based on human characteristics," Simulation Modelling Practice and Theory, vol. 87, pp. 369378, 2018.

[2] X. Z. Zheng, D. Tian, M. Zhang, C. Hu, and L. Tong, "A stairs evacuation model considering the pedestrian merging flows," Discrete Dynamics in Nature and Society, vol. 2019, Article ID 7615479, 2019.

[3] Y. Zhou, T. Wu, G. Zhang, and Z. Fan, "A multistory building evacuation model based on multiple-factor analysis," Advances in Civil Engineering, vol. 2019, Article ID 6585102, 2019.

[4] B. Liu, Z. Liu, D. Sun, and C. Bi, "An evacuation route model of crowd based on emotion and geodesic," Mathematical Problems in Engineering, vol. 2018, Article ID 6585102, 2018.

[5] Y. Shin, S. Kim, and I. Moon, "Simultaneous evacuation and entrance planning in complex building based on dynamic network flows," Applied Mathematical Modelling, vol. 73, pp. 545-562, 2019.

[6] W. Li, J. Zhu, H. Li, Q. Wu, and L. Zhang, "A game theory based on Monte Carlo analysis for optimizing evacuation routing in complex scenes," Mathematical Problems in Engineering, vol. 2015, Article ID 292093, 2015.

[7] Y. Peng, S.-W. Li, and Z.-Z. Hu, "A self-learning dynamic path planning method for evacuation in large public buildings based on neural networks," Neurocomputing, vol. 365, pp. 71-85, 2019.

[8] Y. Jiang, B. Chen, X. Li, and Z. Ding, "Dynamic navigation field in the social force model for pedestrian evacuation," Applied Mathematical Modelling, vol. 80, pp. 815-826, 2020.

[9] X. Guo, J. Chen, Y. Zheng, and J. Wei, "A heterogeneous lattice gas model for simulating pedestrian evacuation," Physica A: Statistical Mechanics and Its Applications, vol. 391, no. 3, pp. 582-592, 2012.

[10] X. Li, F. Guo, H. Kuang, Z. Geng, and Y. Fan, "An extended cost potential field cellular automaton model for pedestrian evacuation considering the restriction of visual field," Physica A: Statistical Mechanics and Its Applications, vol. 515, pp. 47-56, 2019.

[11] J. Wei, W. Fan, Z. Li, Y. Guo, Y. Fang, and J. Wang, "Simulating crowd evacuation in a social force model with iterative extended state observer," Journal of Advanced Transportation, vol. 2020, Article ID 4604187, 2020.

[12] N. Cao, L. Zhao, M. Chen, and R. Luo, "Fuzzy social force model for pedestrian evacuation under view-limited condition," Mathematical Problems in Engineering, vol. 2020, Article ID 2879802, 2020.

[13] M. Mitsopoulou, N. I. Dourvas, G. C. Sirakoulis, and K. Nishinari, "Spatial games and memory effects on crowd evacuation behavior with Cellular Automata," Journal of Computational Science, vol. 32, pp. 87-98, 2019.

[14] V. J. Blue, M. J. Embrechts, and J. L. Adler, "Cellular automata modeling of pedestrian movements," in Proceeding of the Systems, Man, and Cybernetics, vol. 3, IEEE, Orlando, FL, USA, October 1997.
[15] S. Liu, L. Yang, T. Fang, and J. Li, "Evacuation from a classroom considering the occupant density around exits," Physica A: Statistical Mechanics and Its Applications, vol. 388, no. 9, pp. 1921-1928, 2009.

[16] X. Li, Z. Geng, H. Kuang, X. Bai, and Y. Fan, "Effect of dangerous source on evacuation dynamics in pedestrian counter flow," Physica A, vol. 533, Article ID 122047, 2019.

[17] L. Zhang, J. Wang, Q. Shi, and T. Zang, "A new dynamic cellular automaton model for pedestrian evacuation process in stadium," in Proceeding of the 32rd Chinese Control Conference, IEEE, Xi'an, China, July 2013.

[18] X. Yang, B. Wang, and Z. Qin, "Floor field model based on cellular automata for simulating indoor pedestrian evacuation," Mathematical Problems in Engineering, vol. 2015, Article ID 820306, 2015.

[19] Y. Zhang, X. Li, N. Zhu, B. Jia, and R. Jiang, "Evacuation dynamics with smoking diffusion in three dimension based on an extended Floor-Field model," Physica A, vol. 507, pp. 414-426, 2018.

[20] Z. Geng, X. Li, H. Kuang, X. Bai, and Y. Fan, "Effect of uncertain information on pedestrian dynamics under adverse sight conditions," Physica A: Statistical Mechanics and Its Applications, vol. 521, pp. 681-691, 2019.

[21] M. Franovetter, "Threshold models of collective behavior," American Journal of Sociology, vol. 83, no. 6, pp. 1420-1443, 1978.

[22] R. R. McCrae, P. T. Costa, and J. S. Wiggins, Toward a New Generation of Personality Theories: Theoretical Contexts for the Five-Factor Model," the Five-Factor Model of Personality: Theoretical Perspectives, pp. 51-87, The Guilford Press, New York, NY, USA, 1996.

[23] D. Helbing, I. Farkas, and T. Vicsek, "Simulating dynamical features of escape panic," Nature, vol. 407, no. 6803, pp. 487-490, 2000.

[24] J.-H. Wang, W.-Y. Yan, Y.-R. Zhi, and J.-C. Jiang, "Investigation of the panic psychology and behaviors of evacuation crowds in subway emergencies," Procedia Engineering, vol. 135, pp. 128-137, 2016.

[25] M. Shi, E. W. M. Lee, and Y. Ma, "A dynamic impatiencedetermined cellular automata model for evacuation dynamics," Simulation Modelling Practice and Theory, vol. 94, pp. 367-378, 2019.

[26] X. Wang, L. Zhang, Y. Lin, Y. Zhao, and X. Hu, "Computational models and optimal control strategies for emotion contagion in the human population in emergencies," Knowledge-Based Systems, vol. 109, pp. 35-47, 2016.

[27] W. O. Kermack and A. G. McKendrick, "A contributions to the mathematical theory of epidemics," Proceeding of the Royal Society of London, vol. 115, no. 772, pp. 700-721, 1927.

[28] P. S. Dodds and D. J. Watts, "A generalized model of social and biological contagion," Journal of Theoretical Biology, vol. 232, no. 4, pp. 587-604, 2005.

[29] L. Fu, W. Song, W. Lv, and S. Lo, "Simulation of emotional contagion using modified SIR model: a cellular automaton approach," Physica A: Statistical Mechanics and Its Applications, vol. 405, pp. 380-391, 2014.

[30] C. Burstedde, K. Klauck, A. Schadschneider, and J. Zittartz, "Simulation of pedestrian dynamics using a two-dimensional cellular automaton," Physica A, vol. 295, no. 3-4, pp. 507-525, 2001.

[31] L. Zhang, X. Peng, L. Wang, and D. Sun, "Simulation of pedestrian evacuation considering emergency spread and pedestrian panic," Physica A, vol. 522, pp. 167-181, 2019. 
[32] S. Li and H. Niu, "Simulation of pedestrian flow evacuation based on direction fuzzy visual field," Journal of Transportation Systems Engineering and Information Technology, vol. 15, no. 2, pp. 88-95, 2015.

[33] D. Nilsson and A. Johansson, "Social influence during the initial phase of a fire evacuation-Analysis of evacuation experiments in a cinema theatre," Fire Safety Journal, vol. 44, no. 1, pp. 71-79, 2009.

[34] T. Xu, D. Shi, J. Chen, T. Li, P. Lin, and J. Ma, "Dynamics of emotional contagion in dense pedestrian crowds," Physics Letters A, vol. 384, no. 3, Article ID 126080, 2019. 